\title{
Espectrometria de Massas em Geologia Isotópica
}

\author{
Kei Sato \& Koji Kawashita \\ Centro de Pesquisas G eocronológicas - Instituto de Geociências - USP \\ Rua do Lago 562, CEP 05508-080, São Paulo, SP, BRA
}

Keywords: TIMS, SIMS, ICP-MS, Ion Probe, Electron Impact, Mass Spectrometer.

\begin{abstract}
The present paper discusses the main types of mass spectrometers available in 2002 (TIMS, ICP-MS, SIMS and electron impact-MS) with application to isotope geology and especially to geochronology. Brief comments on the principles of operation of the main components, ionization sources, mass analyzers and ion detectors are made. Finally, the performance of the different types of spectrometers is compared.
\end{abstract}

Palavras-chave: TIMS, SIMS, ICP-MS, sonda iônica, impacto de elétron, espectrometria de massa.

\section{RESUMO}

O presente trabalho apresenta os principais tipos de espectrômetros de massas disponíveis em 2002 (TIMS, ICPMS, SIMS, e impacto de elétrons-MS) com aplicação voltada à geologia isotópica, em especial à geocronologia. Breves comentários dos princípios de funcionamento dos principais componentes que constituem o espectrômetro de massa, tais como fontes de ionização, analisadores de massas e detectores de íons, são feitos. Por fim, comparamos os desempenhos de cada tipo de equipamento. 


\section{IN TRO DU ÇÃO}

A datação de eventos geológicos baseada em isótopos radioativos começou com Nier (1939 e 1948) com base nos estudos dos isótopos de $\mathrm{U}$, Th e $\mathrm{Pb}$. Nier observou que dependendo da composição química e da idade, as composições isotópicas de $\mathrm{Pb}$ variavam. Aldrich e Nier (1948) também observaram que as composições isotópicas de $\mathrm{Ar}$ e Ca alteravam devido ao decaimento radioativo de K. Desta forma, nascia a geocronologia com base nestes dois métodos no início dos anos 1950.

Nier modificou o espectrômetro de massa de setor magnético de $180^{\circ}$ para $60^{\circ}$. Esta mudança não só reduziu o peso e o consumo de energia do eletroímã, mas também melhorou a forma de feixe de íons junto ao coletor, bem como na redução do preço do equipamento tornando-o assim acessível para um grande grupo de cientistas, o que antes era privilégio de um seleto número de pesquisadores da física.

Neste trabalho, faremos uma breve descrição dos principais componentes de espectrômetros de massas mais utilizados em geologia isotópica, tais como, NEPTUNE (fig. 1), TRITON (fig. 2), SECTOR, MAT 262, SHRIMP (fig. 3) e outros. Basicamente, os espectrômetros de massa são constituídos de 3 partes principais (fig. 4):

1. fonte de íons;

2. analisador de massas;

3. sistema de detecção.

\section{FO NTE DE ÍONS}

Os espectrômetros de massa trabalham com íons positivos ou negativos de elementos ou espécies moleculares em um sistema de ultra alto vácuo, de $10^{-7}$ a $10^{-9}$ mbar. A ionização de amostra é fundamental, pois é necessário acelerar a massa no analisador que por sua vez faz a deflexão de íons em função da massa, da velocidade e da intensidade de campos elétricos e/ou magnético. Existem várias técnicas de ionização, tais como:
a. termo-ionização;
b. plasma;
c. impacto de elétron;
d. impacto de íon, que serão discutidas a seguir.

\section{Termo-ionização}

As amostras a serem ionizadas são depositadas sobre um filamento metálico previamente limpo. Os filamentos mais utilizados são de Re, Ta, Pt e W e basicamente existem 4 tipos de arranjos geométricos para ionização: filamento simples (fig.5), filamento duplo (fig. 6), filamento triplo (fig. 7) e filamento em forma de canoa (fig. 8). A eficiência de ionização para determinado elemento químico varia em função do tipo de filamento utilizado, ou da função de trabalho $\phi$ ("work function") e do potencial de ionização I do elemento desejado. A função de trabalho é definida na teoria clássica como a energia necessária para que um elétron possa vencer a barreira potencial e ficar livre do metal no vácuo, enquanto que o potencial de ionização é definido como a energia mínima necessária para arrancar um elétron de um átomo. Os espectrômetros que utilizam esta técnica de ionização são denominados de "Thermal Ionization Mass Spectrometer" (TIMS). A eficiência ou proporção de íons $\left(\mu^{+}\right)$relativa a átomo neutro $(\mu)$ é dada pela fórmula: $\left(\mu^{+} / \mu\right) \approx \mathrm{e}^{(} \phi^{-\mathrm{I} / \mathrm{KT})}$ onde $\mathrm{K}$ é constante de Boltzman e T é temperatura absoluta.

A eficiência pode ser alterada se usarmos duplo ou triplo filamento, bem como a forma de deposição. Por exemplo, quando o $\mathrm{Sr}$ é depositado com solução de $\mathrm{H}_{3} \mathrm{PO}_{4}$ sobre o filamento de Ta aumenta-se a eficiência em mais de 10 vezes em relação a qualquer outro tipo de reagente. Do mesmo modo, a deposição de $\mathrm{Pb}$ com a mistura de solução de $\mathrm{H}_{3} \mathrm{PO}_{4}$ e silicagel sobre o filamento de Re aumenta em muito a eficiência de ionização.

A tabela 7 (Apêndice) mostra vários tipos de elementos químicos sólidos de interesse em geocronologia, empregando diversos tipos de arranjos e diferentes tipos de soluções para carregar as amostras sobre o filamento (“sample loading").

\section{Plasma}

A técnica de plasma acoplada indutivamente ("Inductively Coupled Plasma" - ICP) produz com maior eficiência o feixe de íons, que são analisados por meio de espectrometria de massas (ICP/MS) (fig. 9). Uma outra vantagem do ICP em relação às outras formas de ionização é quanto ao mecanismo de introdução de amostra, que se dá através do processo clássico de nebulização ou injeção de átomos ou moléculas liberados por abrasão a laser. Em ambos os casos, a introdução de amostras é operada em 


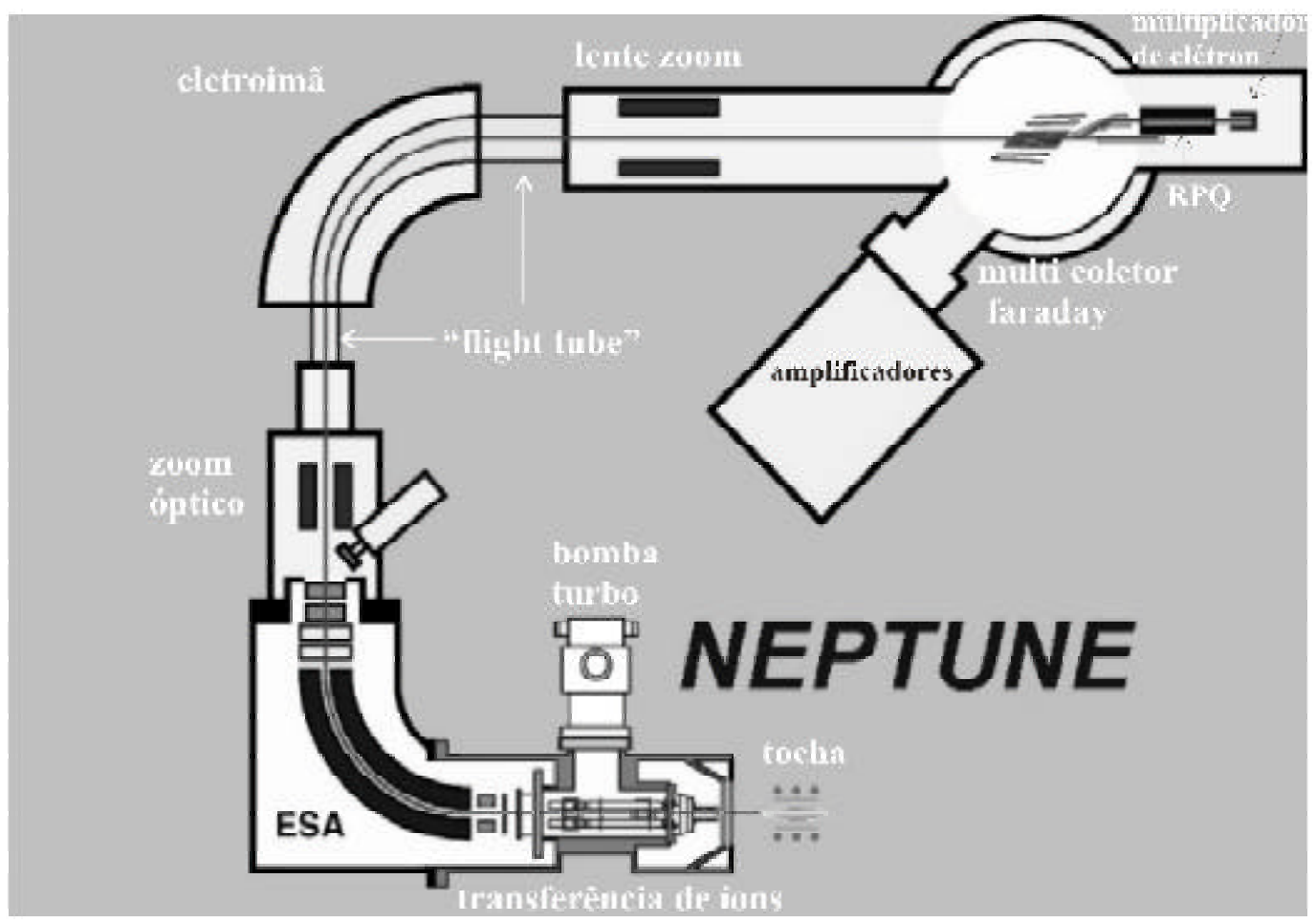

Figura 1. Desenho esquemático do ICP-MS NEPTUNE de Finnigan Mat. RPQ = "retarding potencial quadrupole". Ref.: Finnigan Mat (2001).

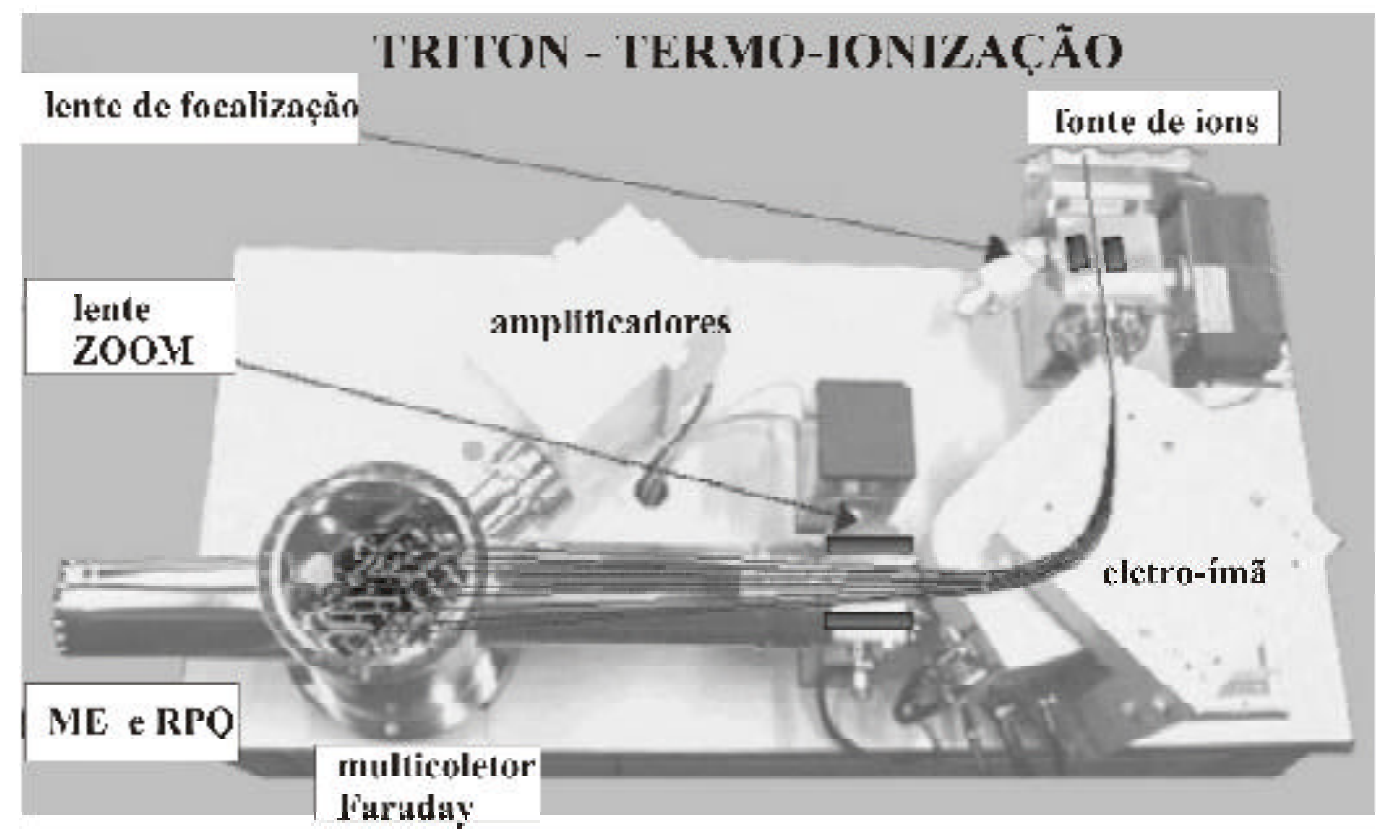

Figura 2. Desenho esquemático do TIMS - TRITO N de Finnigan Mat. RPQ = "retarding potencial quadrupole"; $\mathbf{M E}=$ multiplicador de elétron. Finnigan Mat (2001). 


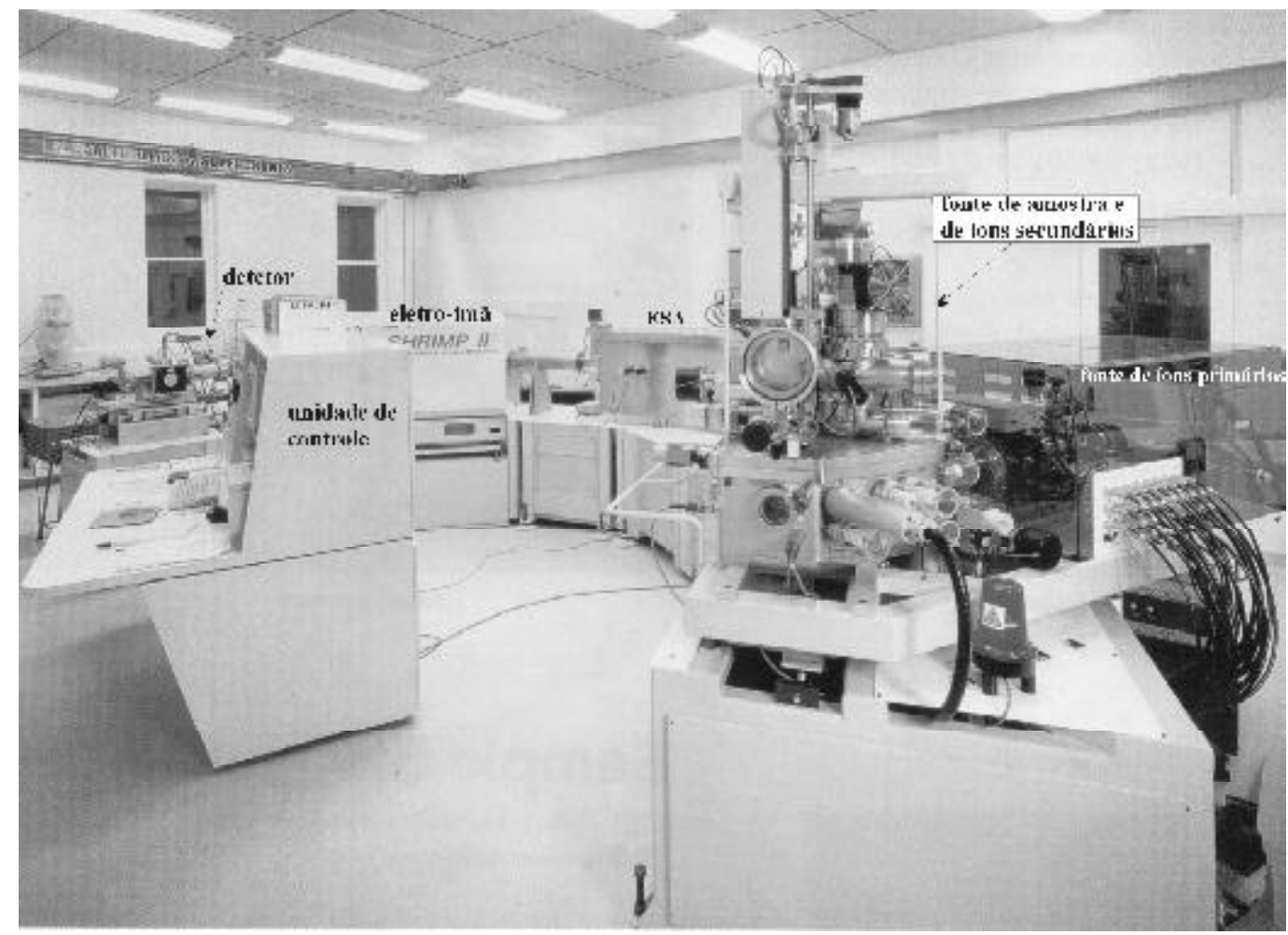

Figura 3. Fotografia do SHRIMP II instalado em Curtin University of Tecnology, Perth - Austrália.

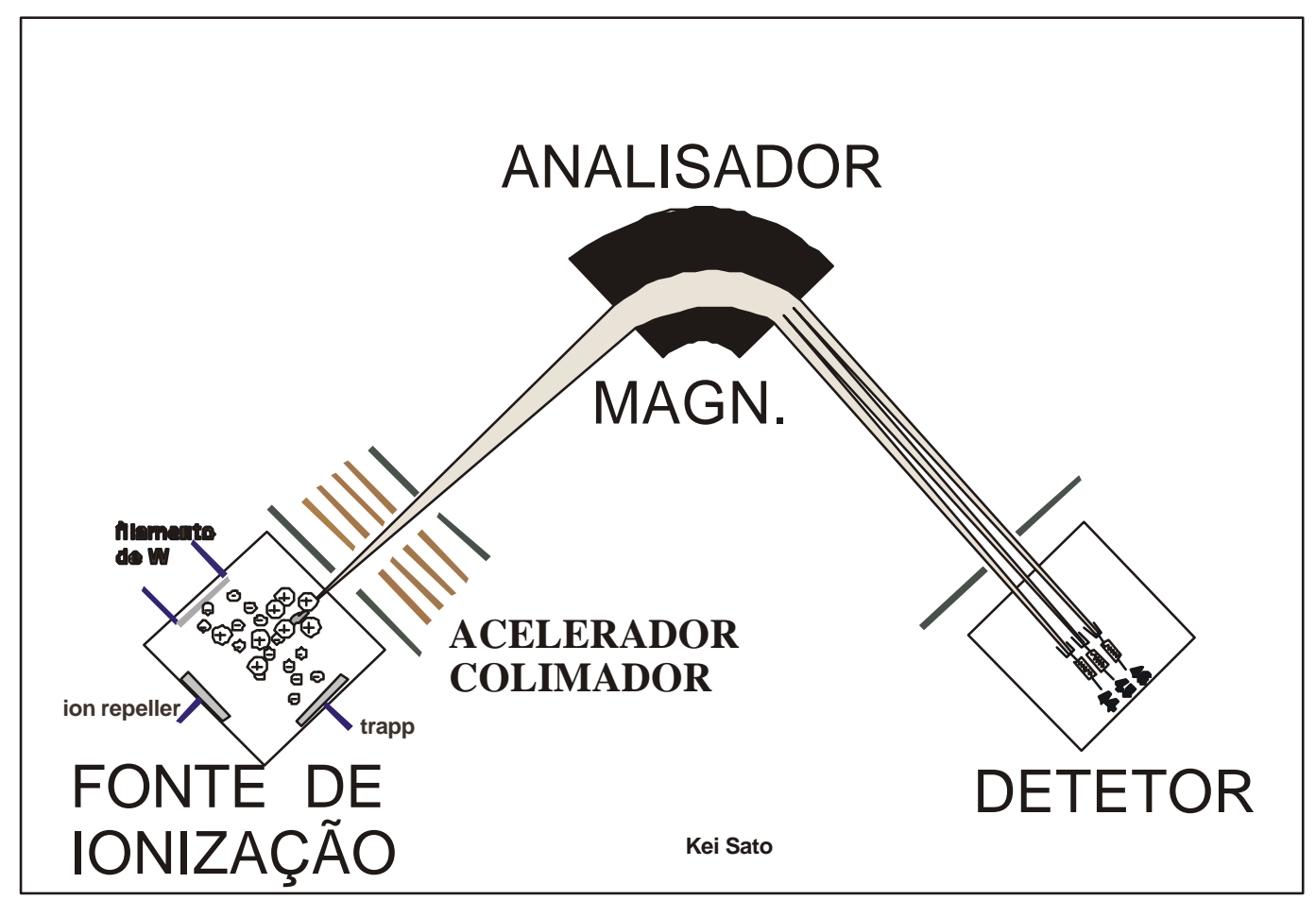

Figura 4. Desenho esquemático do espectrômetro de massa de fonte gasosa. 
condições de pressão ambiental.

Princípio do plasma: um plasma é definido como o alto estado de energia de um gás no qual praticamente todos os átomos e moléculas são ionizados (fig. 9). Para iniciar o protoplasma, um fluxo de gás é semeado com elétrons livres oriundos de uma faísca de alta tensão de enorme potencial capaz de vencer a resistência dielétrica do gás. $\mathrm{O}$ plasma é sustentado por meio de campos elétrico e magnético flutuantes produzidos pelo gerador de onda de rádio freqüência (RF) da ordem de $40 \mathrm{MHz}$ que é produzida dentro de uma bobina de carga ("load coil"). Estes campos acoplam as energias no plasma por meio de aceleração de elétrons livres da periferia para uma zona interna da bobina de carga. Esta zona é conhecida como a região de adição de energia. A energia é então transferida para outra espécie de plasma por meio de colisão. O gás de argônio atinge uma temperatura de 6000 a $8000^{\circ} \mathrm{K}$ na porção central do plasma para um fluxo de 0.7 litros por minuto e a uma pressão de 1.25 bar. Devido ao alto estado de energia do plasma, as moléculas injetadas dentro da tocha são quebradas em seus constituintes elementos químicos que por sua vez, são ionizados.

\section{Introdução de amostras}

A introdução de amostras no ICP pode ser procedida de duas maneiras. No primeiro modo, a solução da amostra é preparada através do sistema químico convencional (1) e no segundo, é usada a técnica de abrasão de amostras sólidas por meio de laser (2).

1. No modo químico, a amostra é dissolvida em reagente químico apropriado, por exemplo, $\mathrm{HNO}_{3}, \mathrm{HCL}, \mathrm{HF}$ etc, e os elementos químicos de interesse são, quando requerido, concentrados em uma coluna de troca iônica. A solução contendo os elementos é injetada na tocha do plasma ("plasma torch"). Os principais componentes do mecanismo para introdução de amostra em solução líquida são sistema de nebulisador, bomba "peristaltic", câmara tipo "spray" e tocha. A seguir, faremos uma breve descrição de cada um destes itens:

Nebulisador - O princípio de operação de um nebulisador pneumático é baseado na quebra de uma corrente de solução de amostra por meio de um fluxo de gás de alta velocidade produzindo "spray" de minúsculas gotas ao redor de $10 \mu \mathrm{m}$ de diâmetro. Uma pequena pressão positiva na câmara de "spray" empurra o aerossol para a tocha;

Bomba peristáltica - A bomba peristáltica é usada para fornecer um fluxo estável de solução de amostra para o nebulisador e para remover alguma solução condensada na câmara de "spray";

Câmara de "spray" - A câmara de "spray" padrão, tipo "Scott Double - Pass", por exemplo, é fabricada em quartzo. Outros materiais tais como PTFE (tipo de teflon) são requeridos se as soluções são altamente corrosivas como o HF, por exemplo. A câmara de "spray" é provida com uma pequena fenda que deixa passar apenas as gotículas menores que $10 \mu \mathrm{m}$ de diâmetro para o plasma, o que corresponde à cerca de $0,1 \%$ da solução total pulverizada. Uma camisa de água refrigerada é utilizada para remover o excesso de água. A remoção deste excesso de água é importante, pois, caso contrário, há uma grande produção de íons de oxigênio dificultando a análise no ICP-MS;

Plasma - No plasma, as moléculas em geral são quebradas e seus elementos químicos são todos ionizados simultaneamente devido à altíssima temperatura do plasma. Por exemplo, no método geocronológico $\mathrm{U}-\mathrm{Pb}$, os isótopos de $\mathrm{Pb}, \mathrm{U}$ e $\mathrm{Tl}\left({ }^{203} \mathrm{Tl},{ }^{205} \mathrm{Tl},{ }^{204} \mathrm{~Pb},{ }^{206} \mathrm{~Pb},{ }^{207} \mathrm{~Pb},{ }^{208} \mathrm{~Pb}\right.$, ${ }^{235} \mathrm{U},{ }^{238} \mathrm{U}$ ) podem ser medidos simultaneamente em um sistema multicoletor. Os isótopos de Tl, no caso, são utilizados para normalizar, ou seja, corrigir as razões isotópicas do $\mathrm{Pb}$ para possíveis efeitos de fracionamento isotópico. Para que estes isótopos sejam detectados simultaneamente, necessita-se de um espectrômetro de massa que tenha uma resolução de massa adequada e que abranja 35 unidades de massa atômica e pelo menos 8 coletores tipo Faraday ou mini contadores de íons baseados em multiplicador de elétrons de dinodo contínuo. A aquisição simultânea das razões isotópicas aumenta em muito a precisão e reprodutibilidade analítica.

2. Abrasão a Laser ("Laser Ablation" - LA) é uma das técnicas de microssonda como é mostrada na fig. 12. Normalmente é utilizado um laser UV de Nd YAG de 266 ou $211 \eta \mathrm{m}$, em geral de forma pulsada. Atualmente existe feixe de laser com comprimento de onda de 193 ๆm (Excimer Lambda) extremamente homogêneo e com o diâmetro que pode variar entre 125 a $5 \mu \mathrm{m}$ (fig. 12). Os componentes abrasados são arrastados/transportados por meio de um fluxo de gás Ar/He (o mesmo utilizado no plasma) para o interior da câmara "spray".

As análises são intercaladas com os padrões para normalizar e/ou corrigir eventuais desvios ("bias") nas medi- 


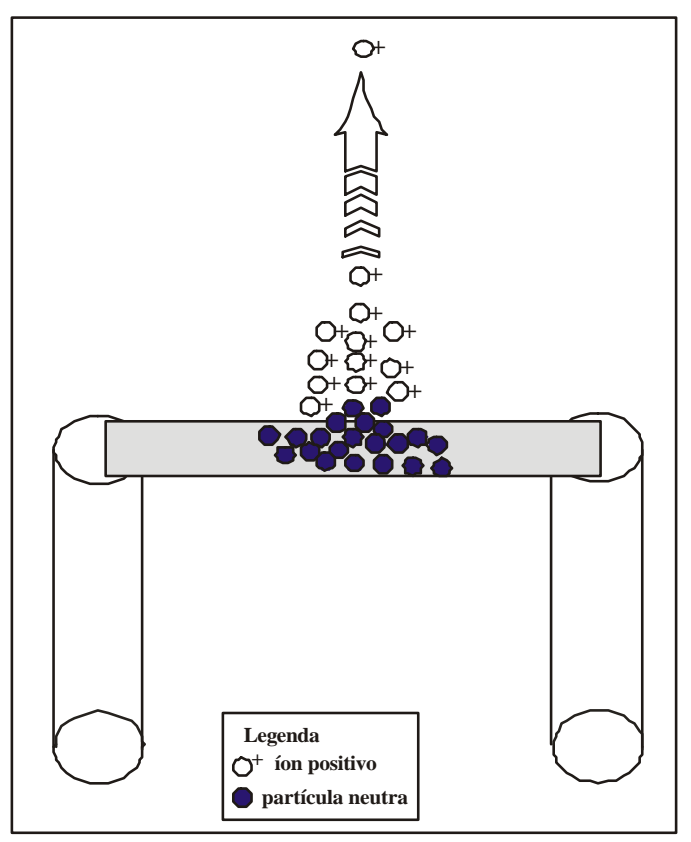

Figura 5. Filamento simples para evaporação e ionização.

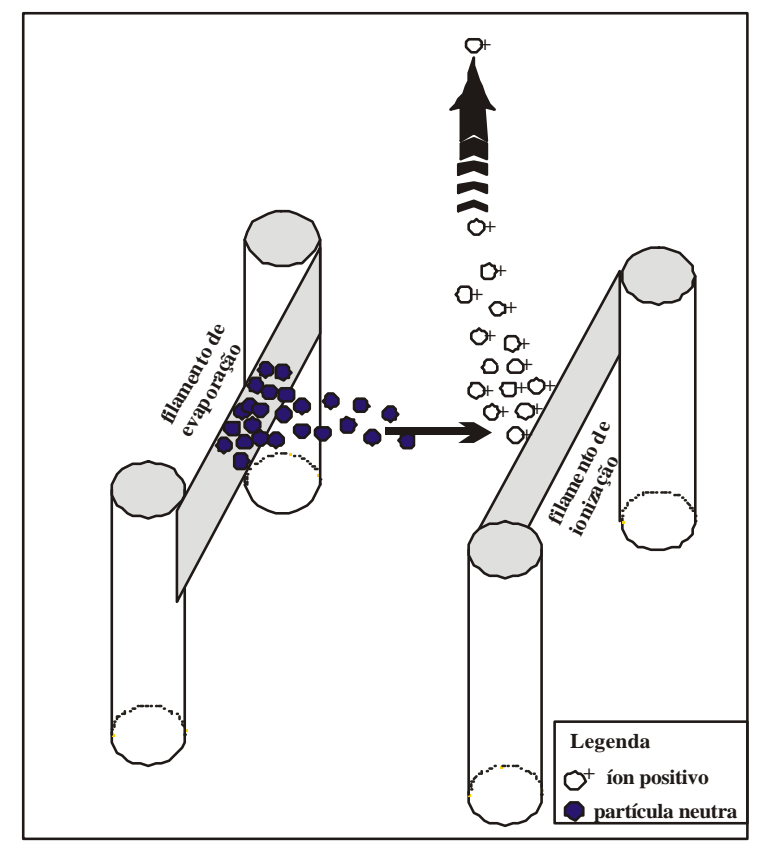

Figura 6. Filamento duplo onde o lado esquerdo é usado para evaporação e o lado direito para ionização.

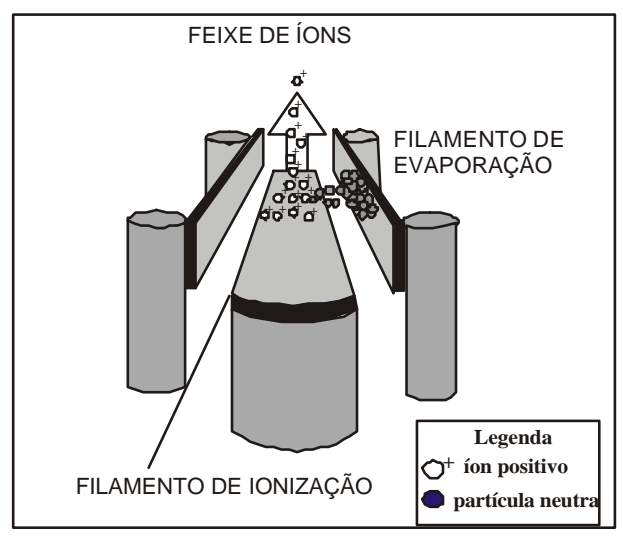

Figura 7. Filamento triplo com o lado direito para evaporação e os lados central e esquerdo para a ionização.

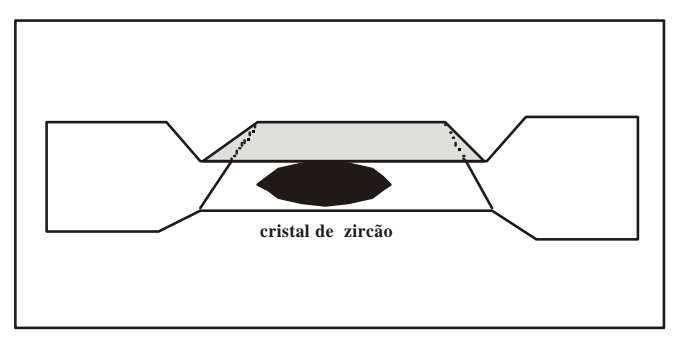

Figura 8. Filamento de rênio na forma de canoa para evaporação e ionização do mono cristal de zircão.

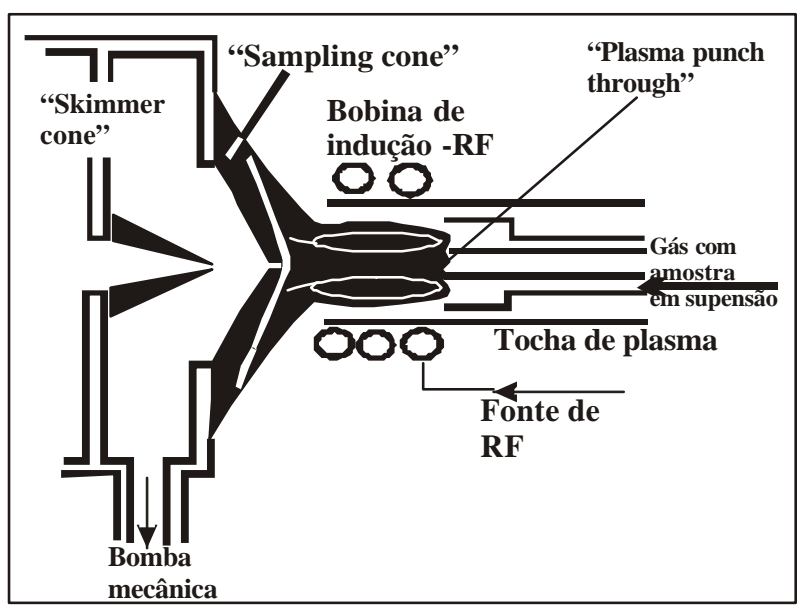

Figura 9. Desenho esquemático da fonte de plasma do IsoProbe - Micromass. Ref.: Micromass (1999). 
das de razões isotópicas das amostras. Opcionalmente para o caso de zircão, os componentes abrasados são injetados simultaneamente com uma solução de spike na câmara de "spray" acoplado ao "plasma torch". Neste caso, é necessário ter uma dupla entrada.

O "Laser Ablation (LA)" acoplado ao "Inductively Coupled Plasma - Mass Spectrometer" (LA-ICP-MS) é conhecido de longa data e são inúmeros os trabalhos publicados envolvendo a datação de zircão pelo método $\mathrm{Pb}-\mathrm{Pb}$. Os primeiros trabalhos empregando LA-ICP-MS para as medidas de razões ${ }^{206} \mathrm{~Pb} /{ }^{238} \mathrm{U}$ e ${ }^{207} \mathrm{~Pb} / 235 \mathrm{U}$ foram efetuadas pela equipe de Hirata \& Nebist (1995), no "Tokyo Institute of Technology” - Tókio - Japão e, posteriormente, outros trabalhos, tais como, Nesbit et al.(1997), Hirata et al. (1998), Li et al. 2001, foram elaborados. A aplicação no método U-Pb introduzindo o "spike" simultaneamente com o material abrasado do zircão, foi testada por Horn $e t$ al. (2000) em Havard University, Cambridge - USA. Os resultados obtidos são comparáveis aos obtidos com Sensitive High Resolution Micro-probe (SHRIMP, fig. 3) que não usa o LA, mas uma sonda de íons primários de oxigênio que são acelerados para abrasar a amostra e produzir íons secundários.

As vantagens do LA-ICP-MS, bem como do SHRIMP, em relação ao sistema convencional na datação de zircão em que se usa o método químico e o TIMS, reside na não necessidade de um laboratório químico super limpo, na rapidez de obtenção dos resultados e, principalmente na possibilidade de efetuar micro-análises localizadas e, portanto, na obtenção de informações adicionais de eventos geológicos impressos no zircão.

\section{Impacto de elétrons}

A técnica de ionização de elementos químicos ou moléculas gasosas é muito diferente da dos sólidos anteriormente discutidos. No processo de ionização dos gases, os elétrons produzidos por filamento de $\mathrm{W}$ aquecido são atraídos e acelerados por uma placa de um campo elétrico positivo, da ordem de 60 a $100 \mathrm{~V}$ (fig. 10), provocando as colisões nos gases introduzidos no espectrômetro e produzindo íons. Os íons produzidos na fonte de íon são repelidos ("ion repeller") por uma tensão positiva e ao mesmo tempo são atraídos e acelerados e colimados por sequiência de placas negativas em relação ao "repeller" na direção do analisador de massas.

\section{Impacto de íons ou sonda iônica ("IO N PRO BE")}

Uma outra maneira de ionizar a amostra é acelerar feixe de íons em alta velocidade contra o alvo. Os espectrômetros de massa CAMECA e SHRIMP utilizam esta tecnologia para efetuar ionização pontual em uma área localizada de um determinado material, por exemplo, o zircão. A sonda ("probe beam") é quase sempre um feixe de íons negativos de oxigênio molecular, $\mathrm{O}_{2}^{-}$. Quando o gás neutro de $\mathrm{O}_{2}$ é injetado na câmara denominada de "duoplasmatron" onde ele é ionizado negativamente na forma de $\mathrm{O}_{2}^{-}$e $\mathrm{O}^{-}$(fig. 11). No SHRIMP somente utiliza-se o íon $\mathrm{O}_{2}^{-}$devido a uma certa vantagem em relação ao $\mathrm{O}^{-}$, isto é, a aceleração de $\mathrm{O}_{2}^{-}$ produz "sputter" e ioniza amostras de diferentes estruturas de uma forma mais uniforme do que o $\mathrm{O}$. Feixes de íons $\mathrm{O}_{2}^{-}$filtrados do $\mathrm{O}^{-}$por um campo magnético perpendicular e acelerados na direção do alvo (amostra), com uma energia de $10000 \mathrm{eV}$, atinge uma velocidade de aproximadamente $884000 \mathrm{Km} / \mathrm{h}$. Os íons são colimados para se obter um feixe com um diâmetro que pode ser escolhido entre 5 a $100 \mu \mathrm{m}$ e, a seguir, acelerados contra a amostra provocando um impacto iônico ("ion crashes") produzindo íons secundários. Os espectrômetros que utilizam esta técnica de ionização são denominados de SIMS ("secondary ion mass spectrometer”). A figura 11, na porção inferior, mostra uma cratera ("spot") com $25 \mu \mathrm{m}$ de diâmetro por $1 \mu \mathrm{mde}$ profundidade, provocada pela abrasão de íons de $\mathrm{O}_{2}{ }^{-} \mathrm{du}-$ rante 15 minutos.

O feixe de íons secundários produzido na fonte de amostra é colimado e acelerado na direção do analisador de massa através do "flight tube" que está em ultra-alto vácuo.

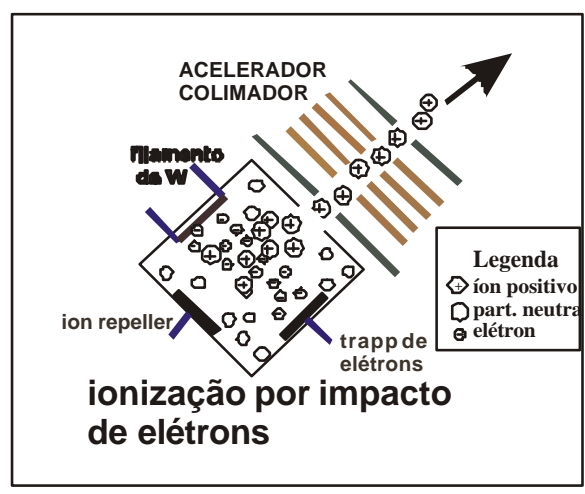

Figura 10. Fonte de ionização por impacto de elétron para gases. 


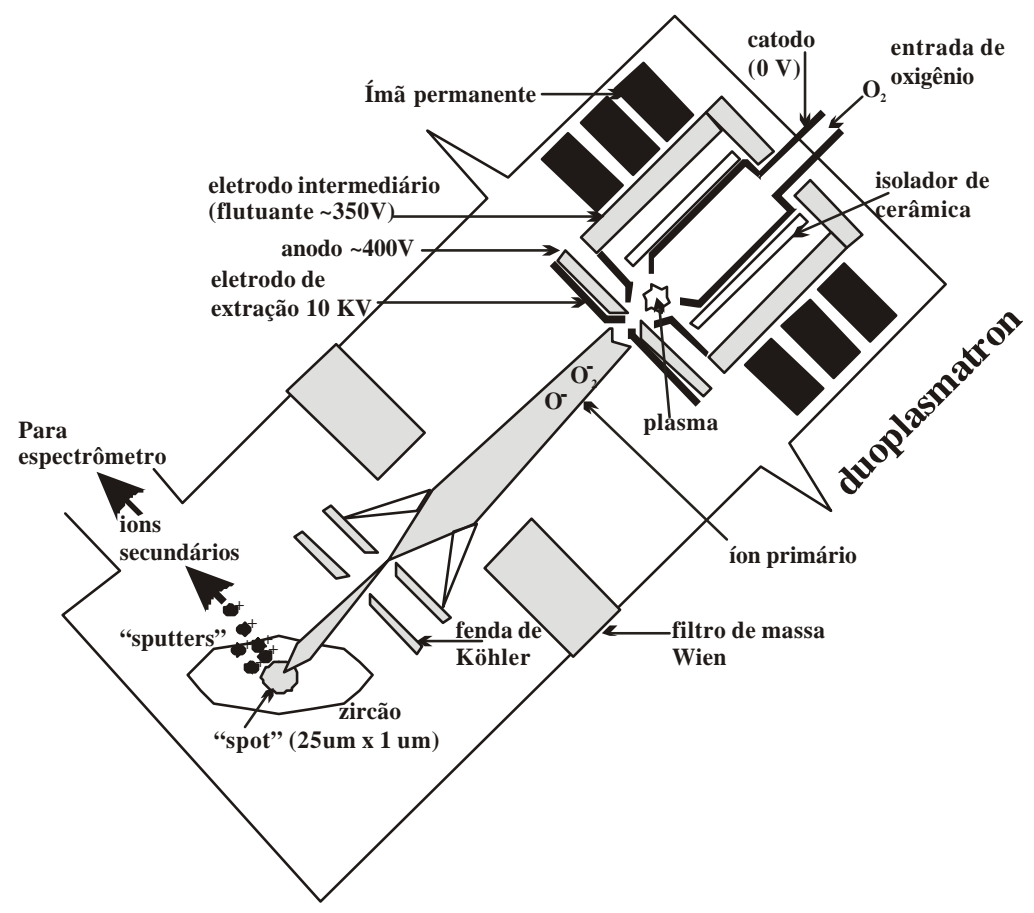

Figura 11. SHRIMP - íons primários (02) e secundários (amostra). Ref.: Willians (1998).

Figura 12. Desenho equemático do caminho óptico do feixe de "laser" (Horn et al. 2000).

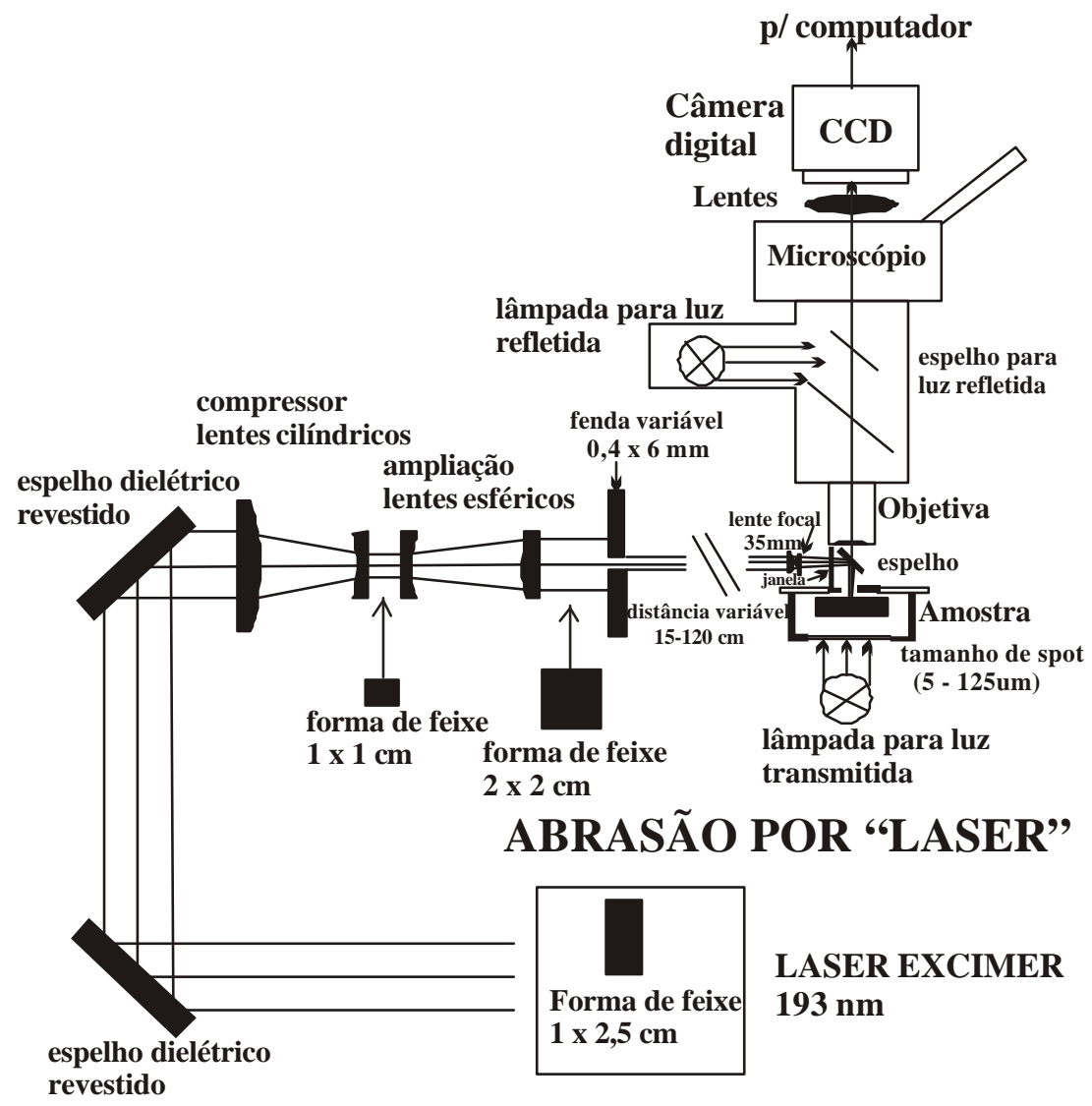




\section{AN ALISADOR DE MASSAS (AM)}

Os íons produzidos em um ICP, filamento termoiônico, impacto de elétrons ou sonda de íons primários são extraídos para dentro de um sistema de lentes que colimam e aceleram os íons para o analisador de massas, que pode ser, na sua versão mais simples, do tipo Quadrupolo (QP) ou por tempo de vôo (TOF - "time of flight"), ou, em versões com maior resolução de massas, Analisador Eletrostático (ESA) e Analisador Magnético (AM). Quando os íons são duplamente defletidos no ESA e AM acabam obtendo uma elevada resolução ( 3000 a 6000) capaz de diferenciar, em certos casos, alguns tipos de isóbaros. Em caso específico do ICP-MS - ISOPROBE da Micromass, a transferência de íons da fonte de plasma para o analisador magnético é baseada em célula de colisão com rádio frequiência conhecida como célula "Hexapole" (fig. 13). As energias dos íons que passam nesta lente são atenuadas por colisões com pequena quantidade de gás He que é introduzido para dentro desta câmara. A colisão de gás de He reduz a energia dos íons de $\sim 30 \mathrm{eV}$ para $\sim 1 \mathrm{eV}$, melhorando muito a característica de transmissão de íons nas lentes de saída do ISOPROBE para o analisador magnético (IsoProbe user's guide - Micromass, 1999). Outros gases reativos, como $\mathrm{H}$, são introduzidos na célula de colisão para reagir com íons interferentes. A seguir, faremos uma breve descrição dos diferentes tipos de analisadores de massas anteriormente referidos:

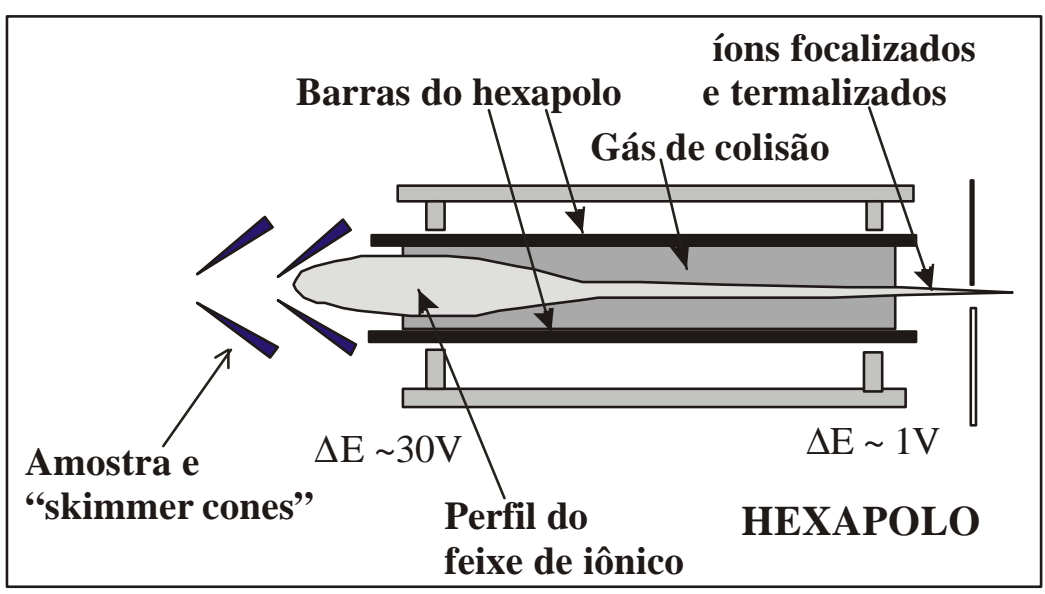

Figura 13. Célula de colisão Hexapolo do IsoProbe - Micromass.

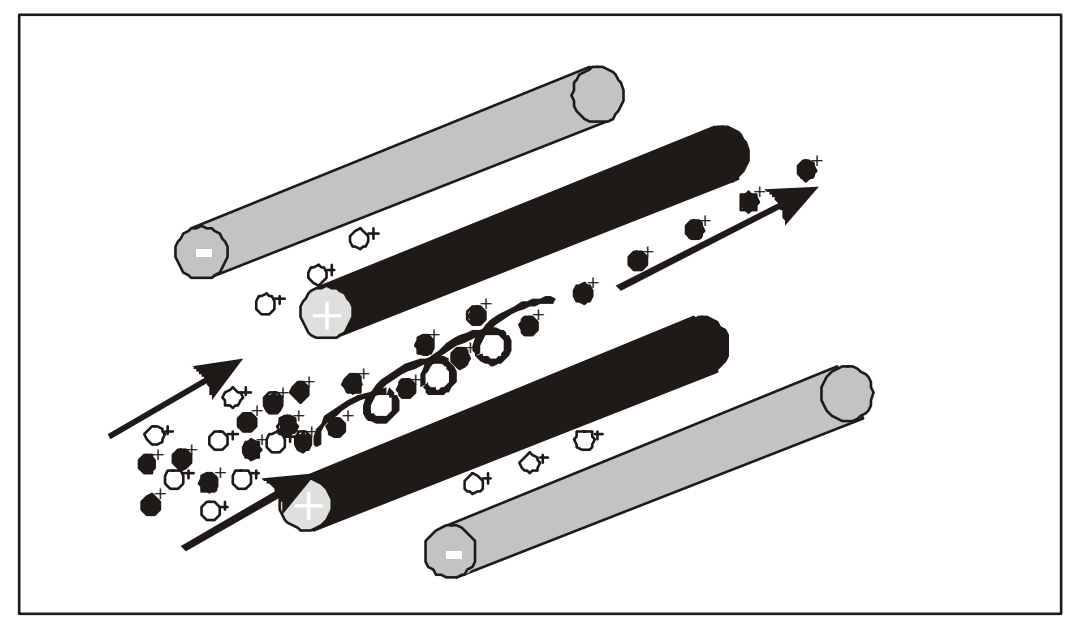

Figura 14. Q UADRUPO LO - ions de círculos fechados foram modulados por campo elétrico flutuante (RF), enquanto que íons de círculos abertos não foram modulados e acabam se chocando contra a parede.
Quadrupolo $(Q P)$ - A figura 14 mostra o desenho esquemático de lentes de quadrupolos. Trata-se de 2 pólos positivos e de 2 pólos negativos posicionados simetricamente e flutuantes na freqüência de rádio $(\mathrm{RF}-\sim 1,2 \mathrm{MHz})$. Os íons que forem acelerados dentro deste campo elétrico mover-se-ão de forma helicoloidal e somente sairão deste setor as massas que forem moduladas pelo RF. A relação entre a massa $(\mathrm{M})$, a freqüência (f), o campo $\gamma_{o}$ e a velocidade (v), que é proporcional à tensão de aceleração, pode ser expressa da seguinte forma: $\mathrm{M}=\mathrm{v} /\left(\mathrm{f}^{2} \gamma_{\mathrm{o}}^{2}\right)$

Tempo de Vôo ou "Time of Flight" (TOF) - Os íons vindos da fonte de plasma são desviados e acelerados ortogonalmente com uma certa freqüência (30 KHz) na direção de "Smartgate ion blanker" (fig. 15). Os íons de mesma massa atingem o "Smartgate" no mesmo intervalo de tempo, enquanto que para os íons mais pesados o tempo gasto para percorrer a distância do acelerador até o "Smartgate" é maior e assim pode-se identificar diferentes massas pela duração de tempo de vôo. No "Smartgate" é focalizado apenas o íon de determinado tempo de vôo que corresponde à massa de interesse, rejeitando-se outros com tempos de vôos diferentes para uma outra direção. Quando o íon passa pelo "ion reflectron" faz aumentar a resolução de massa. $\mathrm{O}$ espectrômetro de mas-

Ref.: Micromass (2000). 


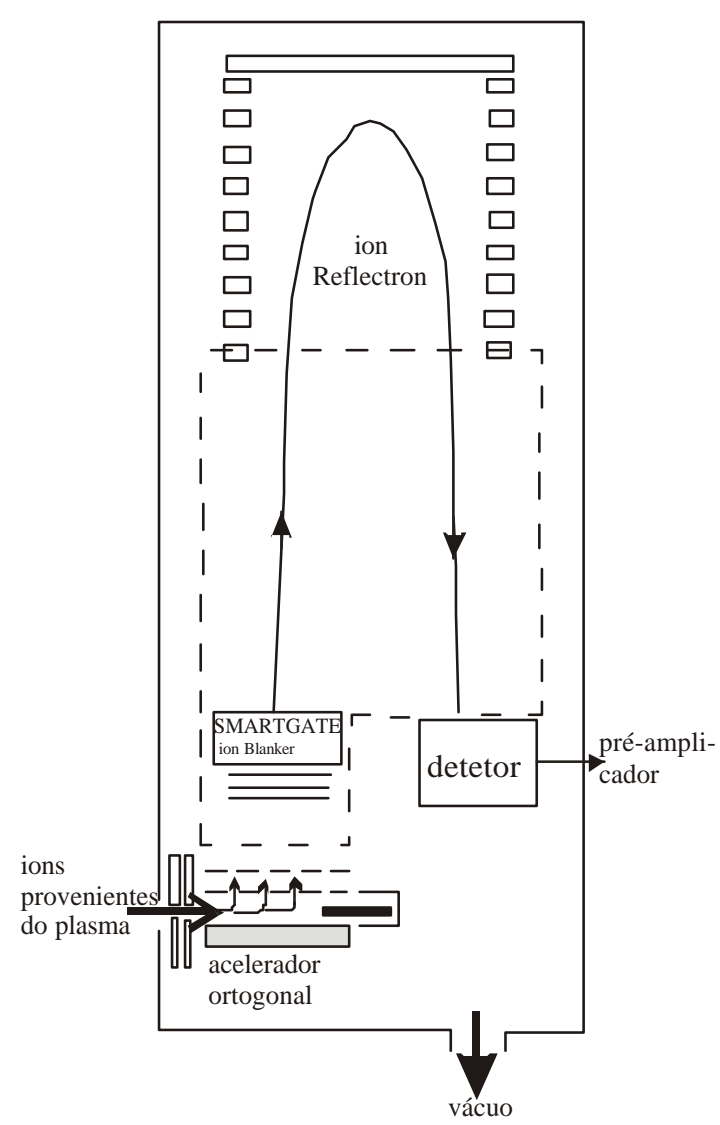

Figura 15. Analisador de massa por tempo de vôo. Ref.: GBC (1998).

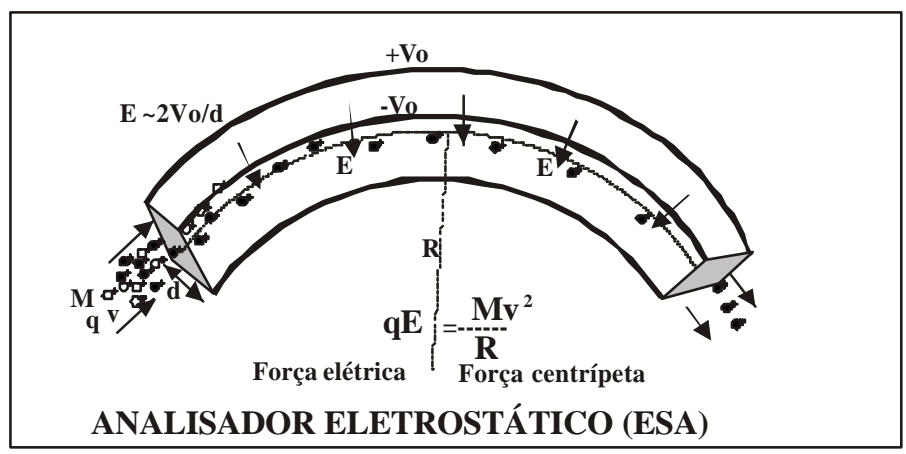

Figura 16. Desenho esquemático do ESA. Somente os ions de círculos fechados têm $\mathrm{Fe}=\mathrm{Fc}$ e conseguem descrever a trajetória curvilínea de raio $R$.

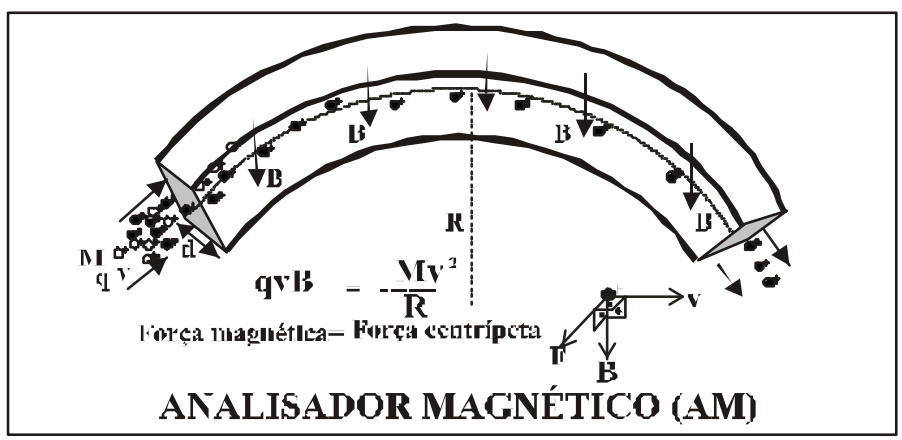

Figura 17. Desenho esquemático do AM. Somente os ions de círculos fechados têm o balanço de força magnética igual a força centrípeta e conseguem descrever a trajetória curva de raio $\mathrm{R}$.

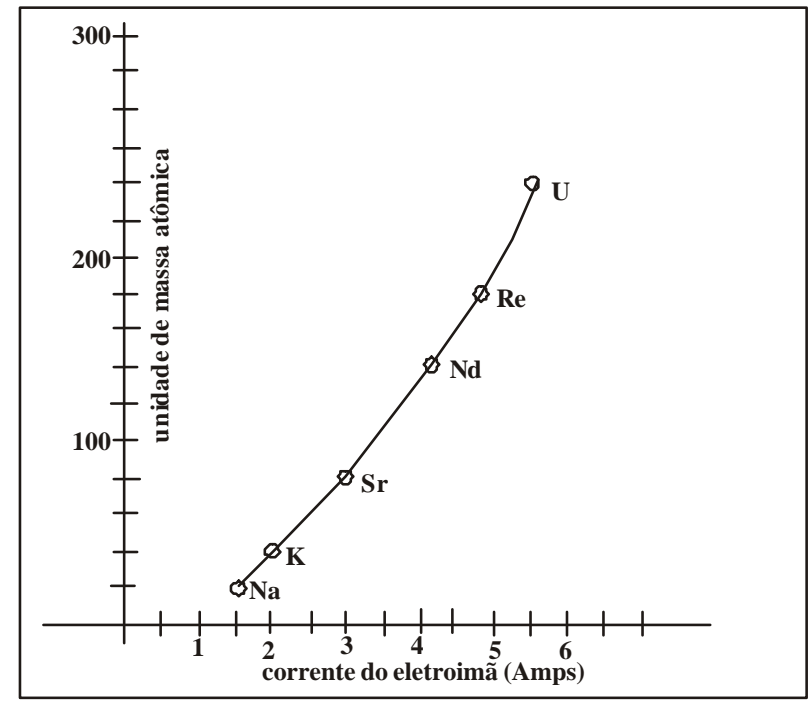

$-66-$
Figura 18. Curva de calibração da massa versus a corrente elétrica aplicada na bobina do eletroimã. A corrente elétrica é diretamente proporcional a intensidade do campo magnético. 
sa OptilMass 8000 da GBC Scientific Equipment emprega esta técnica utilizando o analisador TOF. A resolução de massa deste equipamento na massa 238 é tipicamente igual a 1928 (ver a definição da resolução de massa mais adiante);

"Electro Static Analyzer" (ESA) ou Analisador Eletrostático - os íons quando se movem sob ação de um campo elétrico ficam sujeitos a dois tipos de forças (fig. 16):

$$
\text { força elétrica }\left(\mathrm{f}_{\mathrm{e}}=\mathrm{qE}\right) \text { e força centrípeta }\left[\mathrm{f}_{\mathrm{c}}=\left(\mathrm{Mv}^{2}\right) / \mathrm{R}\right]
$$

onde $\mathrm{M}=$ massa, $\mathrm{v}=$ velocidade, que é proporcional a tensão de aceleração, $\mathrm{R}$ = raio da curvatura do ímã ou eletroímã, $\mathrm{q}=$ carga elétrica e $\mathrm{E}=$ campo elétrico. Os íons somente atravessarão este campo se $\mathrm{F}_{\mathrm{e}}=\mathrm{F}_{\mathrm{c}}$, ou seja:

$$
\begin{aligned}
& \mathrm{F}_{\mathrm{e}}=\mathrm{F}_{\mathrm{c}} \\
& \left(\mathrm{Mv}^{2}\right) / \mathrm{R}=\mathrm{qE} \text { e, portanto, } \\
& \mathrm{R}=\left(\mathrm{Mv}^{2}\right) / \mathrm{qE}
\end{aligned}
$$

O ESA é utilizado nos espectrômetros de massa NEPTUNE, NU INSTRUMENTS, CAMECA, SHRIMP, ELEMENT etc;

"Magnetic Analyzer" (MA) ou Analisador Magnético - Um íon de massa (M) em movimento com velocidade (v) ao atravessar perpendicularmente o campo magnético (B) é submetido à ação de uma força com sentido ortogonal a $\mathrm{B}$ e a v (fig. 17). $\mathrm{O}$ balanço de forças magnéticas $\left(\mathrm{F}_{\mathrm{B}}\right)$ e centrípeta $\left(\mathrm{F}_{\mathrm{c}}\right)$ pode ser escrita da seguinte forma:

$$
\begin{aligned}
& F_{B}=F_{C} \\
& q v B=\mathrm{Mv}^{2} / R, \text { ou seja, } \\
& M=B(R q / v)
\end{aligned}
$$

Onde $\mathrm{R}$ = raio de curvatura e q é carga elétrica. Portanto, como R e q são constantes, e se fixamos a velocidade $\mathrm{v}$ (proporcional à tensão de aceleração), devemos ajustar o campo $\mathrm{B}$ diretamente proporcional à massa $\mathrm{M}$ para que este íon possa descrever a sua trajetória curvilínea de raio R. A equação acima pode ser rescrita utilizando-se a energia cinética $(\mathrm{W})$ de massa $\mathrm{M}$ e de velocidade v:

$$
\mathrm{W}=\mathrm{Mv}^{2} / 2 \text { ou } \mathrm{v}^{2}=2 \mathrm{~W} / \mathrm{M} ; \text { portanto }
$$

$\mathrm{M}=\mathrm{q}^{2} \mathrm{~B}^{2} \mathrm{R} / 2 \mathrm{~W}$, onde $\mathrm{W}$ é igual à energia de aceleração.
Considerando a tensão de aceleração igual a $10 \mathrm{Kvolts}$ e a carga q igual a $1,6 \times 10^{-19}$ Coulomb, então a energia W é igual a:

$$
\mathrm{W}=10 \mathrm{keV}=1,6 \times 10^{-15} \text { Joules. }
$$
forma:

O campo B, portanto, pode ser escrito da seguinte

$$
B=\left\{2 M W / q^{2} R^{2}\right\}^{1 / 2}
$$

Por exemplo, uma massa 206 (massa exata $=205,9745$ ), uma vez ionizada e acelerada com uma energia de $1,6 \times 10^{-15}$ Joules, descreve uma trajetória curvilínea de raio $\mathrm{R}$ igual a 1,000 m se estiver sob a ação de um campo magnético B igual a 0,20674 T ou 2067,4 G.

A grande maioria dos espectrômetros de massas é dotada de analisador magnético, tais como, MAT 262, NEPTUNE, TRITON, SECTOR 54, SECTOR, VG 354, DELTA E, DELTA PLUS, GEO - 20-20, NUCLIDE, PRISMA, SIRA, SHRIMP, CAMECA, ISOPROBE, NU INSTRUMENTS, ELEMENT2 etc.

A figura 18 mostra a correlação entre a massa $\mathrm{M} \mathrm{e}$ campo B. De posse desta curva de calibração, os parâmetros da parábola são armazenados na memória do computador. Para uma análise de rotina basta informar ao microcomputador a massa desejada que este irá ajustar de forma "on line" a intensidade do campo magnético B. A resolução do espectrômetro de massa depende em muito do raio de curvatura, da geometria do ímã ou eletroímã, das lentes ESA e QP. A resolução de massa é definida da seguinte forma:

$\mathrm{R}=\mathrm{M} / \Delta \mathrm{M}$ onde $\mathrm{M}=$ massa nominal no centro do pico e $\Delta \mathrm{M}$ é a largura do pico a determinada altura.

Em uma análise de zircão medimos os isótopos de $\mathrm{Pb}$ de massas 204, 206, 207 e 208. Uma das interferências do $\mathrm{Pb}$ é o ${ }^{176} \mathrm{Hf}^{16} \mathrm{O}_{2}$ com massa total de 208 . No entanto, as massas verdadeiras de ${ }^{208} \mathrm{~Pb}$ e ${ }^{176} \mathrm{Hf}^{16} \mathrm{O}_{2}$ diferem de 0,0454 conforme mostrado abaixo:

\begin{tabular}{|l|l|l|l|}
\hline & ${ }^{176 \mathrm{Hf}^{16} \mathrm{O}_{2}}$ & ${ }^{208} \mathrm{~Pb}$ & $\Delta \mathrm{M}$ \\
\hline Massa verdadeira & 207,91312 & 207,9766 & 0,0454 \\
\hline
\end{tabular}

Para que ${ }^{208} \mathrm{~Pb}^{176} \mathrm{Hf}^{16} \mathrm{O}_{2}$ não interfiram entre si é necessário que o espectrômetro tenha resolução de massa 
de 4600, ou seja:

$$
\mathrm{R}=207,9766 / 0,0454=4580
$$

Para o caso do SHRIMP, o feixe de íons secundários produzido na fonte de amostra é colimado e acelerado no interior do tubo analisador que está em ultra-alto vácuo. A tríplice combinação de lentes iônicas constituídas de quadrupolo, que ajusta o feixe para uma máxima transmissão, uma câmara analisadora eletrostática (ESA) e analisador de massas de setor magnético leva a uma alta resolução em massa da ordem de 5000 - 10000, capaz de distinguir alguns tipos de isóbaros interferentes. Os íons podem ser detectados utilizando o mono coletor copo de "Faraday", no caso de sinais elevados ou sistema como multiplicador de elétrons/contador de íons no caso de sinais de baixa intensidade. Os SHRIMP I e II são equipados com apenas um coletor, portanto, faz-se o ajuste de intensidade do campo magnético na forma de "peak jump" para que determinada massa ou outras massas sejam defletidas e detectadas no mesmo coletor. Do mesmo modo, o ICPMS NEPTUNE pode atingir a resolução acima, mas a intensidade do feixe diminuirá de forma drástica, pois é necessário estreitar as fendas de entrada e de saída dos feixes. Alguns tipos de interferências, tais como, ${ }^{187} \mathrm{Os} \mathrm{e}$ ${ }^{187} \mathrm{Re},{ }^{87} \mathrm{Rb}$ e ${ }^{87} \mathrm{Sr}$ e ${ }^{176} \mathrm{Hf} \mathrm{e}{ }^{176} \mathrm{Lu}$ ainda não são resolvidas. Para estes tipos de isóbaros a única maneira de corrigir as interferências seria a de isolar os elementos interferentes através de colunas de troca iônica ou através de correções matemática apropriadas, como exemplificado abaixo:

$$
{ }^{187} \mathrm{Os}={ }^{187}(\mathrm{Re}+\mathrm{Os})_{\mathrm{med}}-1.67388^{185} \mathrm{Re} \text { : considerando cons- }
$$
tante a razão ${ }^{187} \mathrm{Re} /{ }^{185} \mathrm{Re}=1.67838$

${ }^{87} \mathrm{Sr}={ }^{87}(\mathrm{Sr}+\mathrm{Rb})_{\mathrm{med}}-0.3857{ }^{85} \mathrm{Rb}$ : considerando constante a razão ${ }^{87} \mathrm{Rb} /{ }^{85} \mathrm{Rb}=0.3857$

${ }^{176} \mathrm{Hf}={ }^{176}(\mathrm{Hf}+\mathrm{Lu})_{\mathrm{med}}-0.02659{ }^{175} \mathrm{Lu}$ : considerando constante a razão ${ }^{176} \mathrm{Lu} /{ }^{175} \mathrm{Lu}=0.02659$

Em ICP-MS existe uma outra maneira de eliminar as interferências, por exemplo, a separação dos isótopos de $\mathrm{Rb}$ e do $\mathrm{Sr}$ pode ser feita através da reação química injetando-se gases reativos $\mathrm{NO}_{2}+\mathrm{CH}_{4}$ na câmara de colisão do ICP:

$\mathrm{Sr}^{+}+\mathrm{N}_{2} \mathrm{O} \rightarrow \mathrm{SrO}^{+}+\mathrm{N}_{2}:$ a reação é espontânea e exotérmica.

$\mathrm{Rb}^{+}+\mathrm{N}_{2} \mathrm{O} \rightarrow \mathrm{RbO}^{+}+\mathrm{N}_{2}$ : a reação é endotérmica e não é espontânea.
Portanto, após a reação química o Rb é analisado na forma metálica $\left(\mathrm{Rb}^{+}\right)$enquanto que o $\mathrm{Sr}$ é analisado na forma de óxido $\left(\mathrm{SrO}^{+}\right)$.

Em caso de TIMS o Rb é separado do Sr por diferença de temperatura, isto é, o $\mathrm{Rb}$ se evapora em temperatura mais baixa do que o Sr. O Sm pode ser separado do Nd durante a deposição destes elementos sobre o filamento de tântalo efetuando-se a seguinte reação química:

$$
\begin{aligned}
& \mathrm{Sm}+\mathrm{Nd}+\mathrm{H}_{3} \mathrm{PO}_{4}+\text { calor (filamento de Ta ao rubro) } \rightarrow \\
& \mathrm{Sm}+\mathrm{NdO}+\mathrm{SmO} .
\end{aligned}
$$

Praticamente $95 \%$ de Nd são oxidados contra menos que $10 \%$ de Sm.

Uma das vantagens do SHRIMP em relação ao LAICP-MS reside no fato da abrasão feita pelos íons primários na amostra ser relativamente pequena em termos de profundidade envolvida, que é da ordem de 2 a $3 \mu \mathrm{m}$, mas suficiente para fazer uma micro análise.

\section{SISTEMA DE DETECÇÃO}

As versões mais atualizadas de ICP-MS e TIMS são dotadas de um sistema de detecção simultânea de íons, tais como, multicoletores Faraday ou multiplicador de elétrons acoplado ao contador de íons, detector "Daly" (usado nos espectrômetros Micromass), ou combinação dos mesmos, além do sistema detector MSC ("multi channel scaler") utilizado no ICP-MS da Varian.

\section{Copo de Faraday}

As figs. 19 a, b e c mostram os desenhos esquemáticos de copo de Faraday de alta eficiência. O fluxo de íons que chega continuamente ao copo de "Faraday" é neutralizado por elétrons provenientes da terra que sobem através de um resistor $\mathrm{R}$ de valor conhecido que varia entre $10^{7}$ a $10^{12} \Omega$ (fig 19b). Se fluir uma corrente de elétron de forma proporcional ao fluxo de íons sobre o resistor R, então podemos medir tensão em volts. A tensão medida é diretamente proporcional à concentração destes isótopos contidos na amostra. Os multicoletores (fig. 19c) aumentam em muito a precisão analítica, pois independe da instabilidade do feixe na fonte de íons, mas a reprodutibilidade e exatidão são dependentes da calibração dos ganhos dos 
amplificadores (ver mais adiante).

\section{Contador de íons e multiplicador de elétrons}

Quando os íons que chegam aos detectores são muito baixos $\left(<10^{-14} \mathrm{~A}\right)$ podem ser contados utilizando-se o clássico contador de pulsos. Para tanto, utiliza-se o detector "Daly" (ver mais adiante) ou o multiplicador de elétrons, conforme o desenho esquemático mostrado na figura 20. O multiplicador de elétron consiste de conjunto de dinodos à base de $\mathrm{Cu}$-Be polarizados com alta tensão entre 1 e $3 \mathrm{KV}$. Quando um íon colide com alta velocidade sobre o primeiro dinodo arranca uma certa quantidade $\mathrm{X}$ de elétrons. A quantidade $X$ de elétrons sobre a primeira célula depende da tensão de polarização dos dinodos. Estes X elétrons, quando incidem sobre o segundo dinodo, aumentam em $\mathrm{X}^{2}$ e estes $\mathrm{X}^{2}$ elétrons, quando incidem na terceira célula, aumentam em $\mathrm{X}^{3}$. A quantidade final de elétrons amplificados se dá na forma de progressão geométrica com ganho de $\mathrm{X}^{\mathrm{N}}$ onde $\mathrm{N}$ é o número total de dinodos. Um contador de pulso tem sua limitação, isto é, se entrarem 2 pulsos quase que simultaneamente no contador somente será registrado uma contagem. Este limite de tempo mínimo entre uma contagem e a outra é conhecido como tempo morto e é da ordem de 30 nano segundos (fig. 20). A grande maioria dos espectrômetros é equipada apenas com um sistema de multiplicador de elétrons/contador de íons porque ele ocupa um espaço físico muito grande, exceto o NU Instruments que é dotado de três conjuntos.

\section{Detector "Daly"}

O "Daly" é um sistema detector de íons positivos utilizado com exclusividade nos equipamentos Micromass. Os íons positivos são acelerados e desviados por meio de alta tensão negativa $(-25 \mathrm{KV})$ a um catodo aluminizado (Daly), (fig. 21). No impacto destes íons no catodo são produzidos 8 elétrons secundários, que são imediatamente repelidos na direção oposta onde se acha um cintilador à base de fósforo. Estes elétrons emitidos são convertidos em pulsos de luz no cintilador e são acusados pelo eletrodo fotossensível da válvula fotomultiplicadora que fica externamente do "flight tube", porém acoplada através de um visor especial. O fator de ganho de amplificação é ajustado para dar 1 pulso de íon gerado na fonte de amostra para 100 contagens na válvula fotomultiplicadora. Por ser gran- de (problema de espaço físico), os espectrômetros têm instalado apenas um "Daly". Para contornar essa deficiência são instalados mini contadores de íons (multiplicador de elétrons de dinodo contínuo, fig. 22 a e b). Porém, por serem pequenos, sua vida útil é curta comparada ao detector "Daly".

\section{Lente de retardo}

Quando um íon colide com partículas de gás neutro, durante seu vôo da fonte de amostra até o coletor, sofre espalhamento e conseqüentemente diminuição de sua energia cinética aumentando, deste modo, o "background". Lentes de retardamento eliminam estes tipos de íons que sofreram as colisões. Os espectrômetros de massa da Finnigan Mat e da Micromass oferecem como opção os filtros RPQ e WARP, respectivamente. No espectrograma da fig. 23a mostra a variação da linha de base na direção do pico ${ }^{235} \mathrm{U}$ devida à interferência do "tail" da massa ${ }^{238} \mathrm{U}$ super intensa, e na fig. 23b, o mesmo espectro após a aplicação do filtro WARP. Também para análise dos isótopos de ${ }^{230} \mathrm{Th}$, as lentes de retardo têm sido freqüentemente utilizadas devido ao alto teor dos isótopos de ${ }^{232} \mathrm{Th}$ que interfere sobre os isótopos 230Th de baixíssima quantidade. O padrão de Th do laboratório “Open University” possui a razão ${ }^{232} \mathrm{Th} /{ }^{230} \mathrm{Th}$ da ordem de 165000 (Finnigan Mat, 1995).

\section{Multicoletores e lente zoom}

Na grande maioria dos espectrômetros construídos anteriormente a 2000, os coletores eram pré-ajustados internamente para determinado conjunto de isótopos, tais como, DELTA E, DELTA PLUS, GEO 20-20, SIRA, PRISMA, MAT 230, IsoPrime etc, ou então os coletores eram móveis e ajustados externamente através de "bellows" para que todos os isótopos a serem medidos entrassem simultaneamente $100 \%$ nos detectores. A técnica de coletores, móveis tal como existente nos espectrômetros MAT 261, MAT 262, VG 354, SECTOR 54, SECTOR etc, constituíram em um grande avanço na espectrometria propiciando maior precisão, bem como rapidez nas medidas de razões isotópicas. A partir de 1998, houve uma inovação nos ajustes de entrada dos íons nos coletores, ou seja, os feixes passaram a ser ajustados eletronicamente através de lente ZOOM em frações de segundos, sem a necessidade de mover os coletores. Nos espectrômetros NU Instruments, 


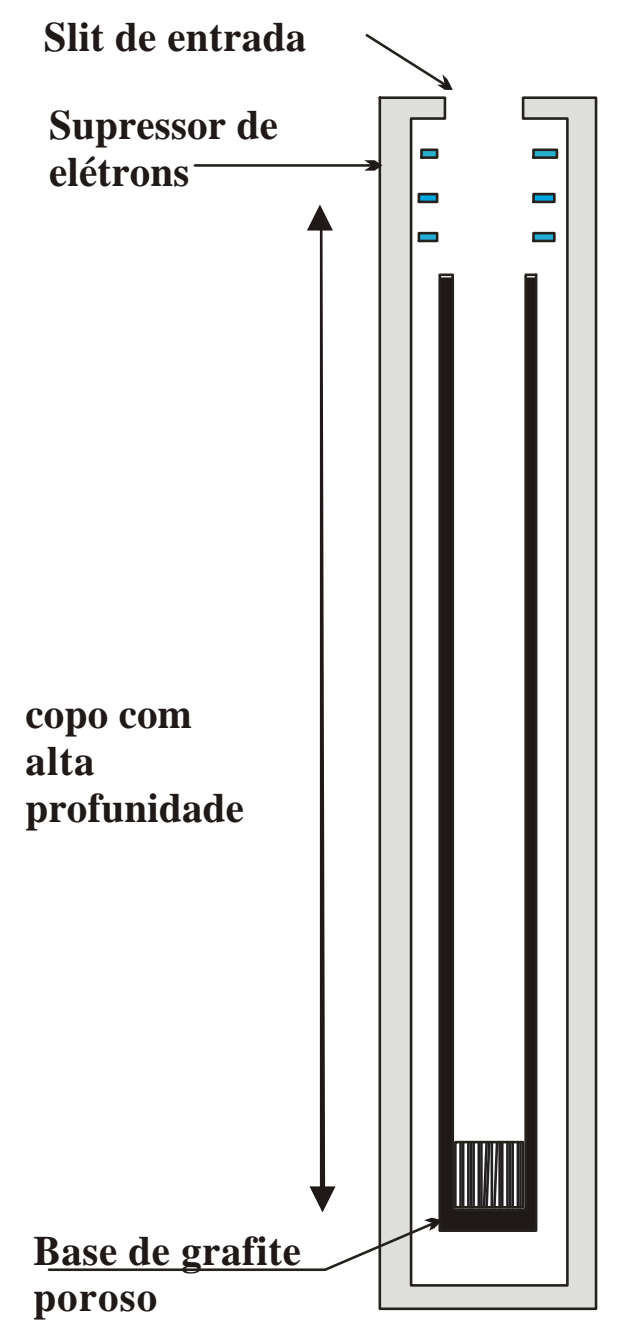

Figura 19a. Coletor Faraday de alta eficiência. (VG Sector 54).

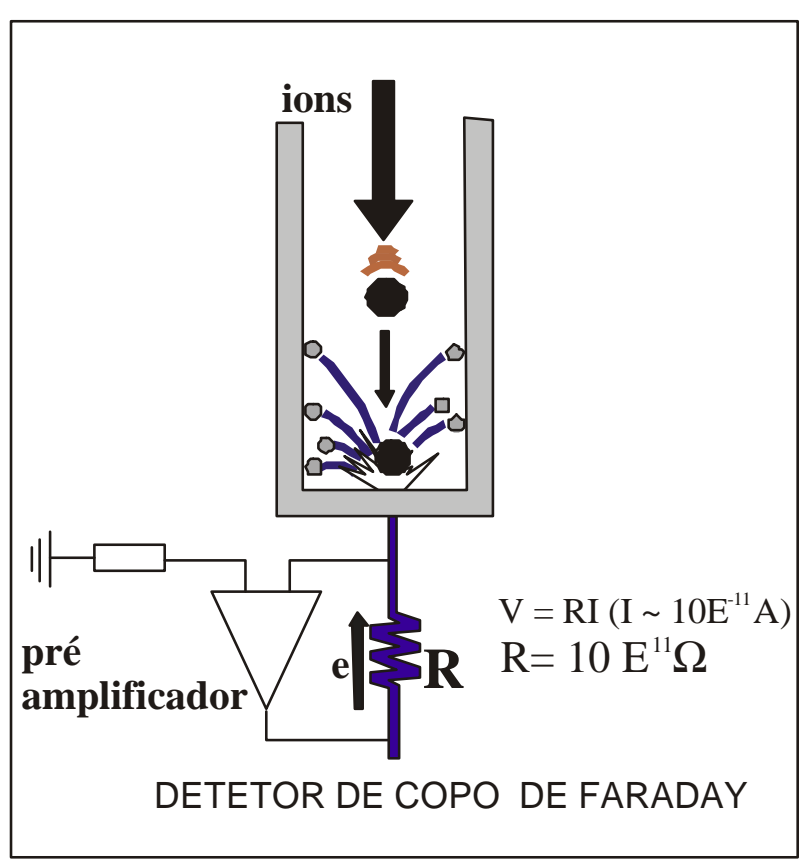

Figura 19b. Detetor de copo de Faraday com préamplificador. Os elétrons fluem através da resistência $R$ neutralisando os íons.

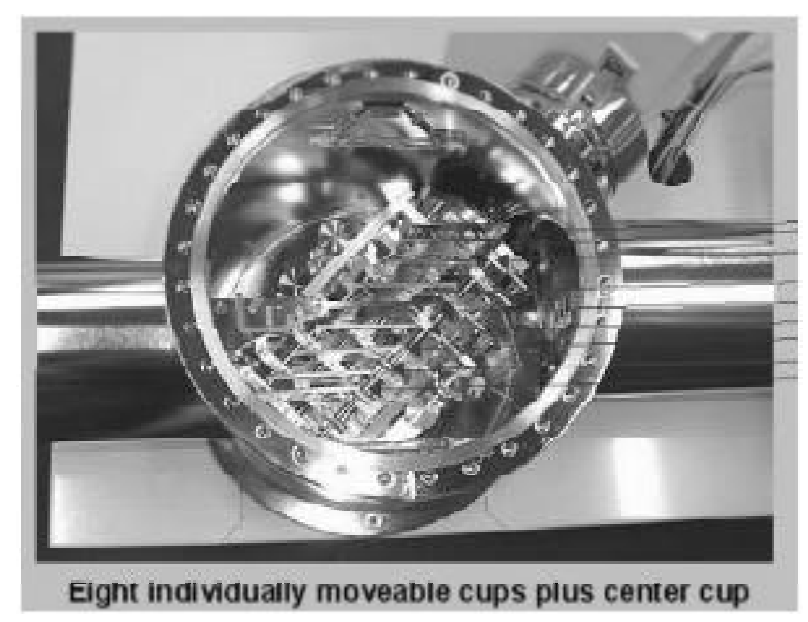

Figura 19c. Fotografia do multicoletor TRITO N/NEPTUNE. Existem 8 coletores móveis e um central fixo localizado fora deste conjunto. Ref.: Finnigan Mat (2001). 


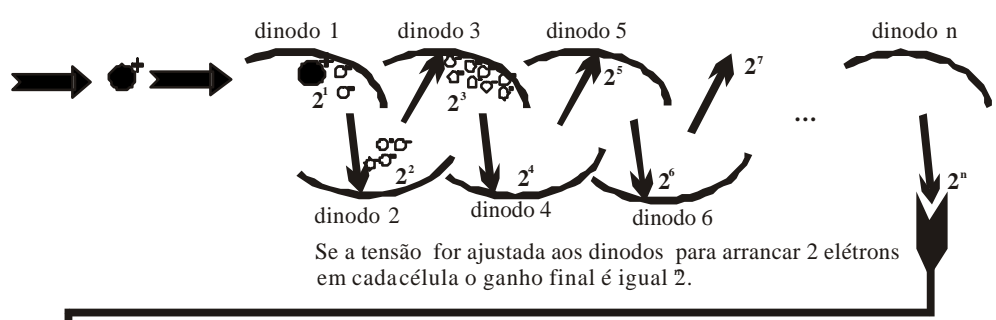

Figura 20. Multiplicador de elétrons acoplado ao contador de íons.

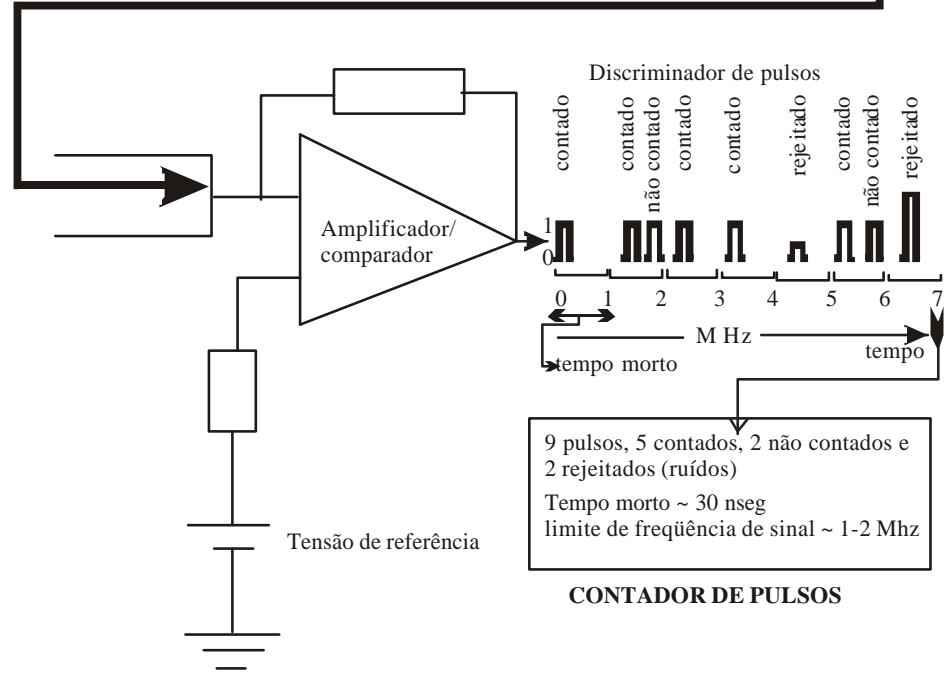

Figura 21. Princípio do detetor "Daly". Informações técnicas baseadas no catálogo do Micromass. Ref.: VG Isotech (1995). 
por exemplo, os isótopos de $\mathrm{Nd}, \mathrm{Pb}$ e $\mathrm{U}$ podem ser medidos na forma sequiencial com extrema rapidez, pois não necessitam mover os coletores. Os espectrômetros TRITON e NEPTUNE podem medir todos os isótopos de $\mathrm{UO}_{2}$ usando a mesma configuração dos coletores de $\mathrm{U}$ sem a necessidade do reajuste mecânico das posições dos coletores (fig. 1).

\section{Calibrações dos amplificadores}

Para calibrar individualmente os amplificadores de copo de Faraday convencionalmente utiliza-se uma fonte de corrente constante para medir o fator de amplificação de cada detector. Para medir as eficiências dos detectores e calibrar o ganho relativo entre o amplificador de copo de Faraday e o sistema multiplicador de elétron/contador de íons utiliza-se a fonte de feixe de íon super constante. Para minimizar os erros de calibrações, a intensidade iônica dos diferentes isótopos é medida simultaneamente em diferentes amplificadores em três seqüências distintas, de forma a cancelar os fatores de ganho e eficiência. Esta técnica de análise é conhecida como sistema de medida no modo dinâmico combinado ao modo estático usando o multicoletor. Já nos equipamentos de TRITON e NEPTUNE, os amplificadores são calibrados utilizando-se uma mesma corrente que é aplicada em todos os outros amplificadores na forma sequiencial, chaveando um conjunto de "relays" e eliminando assim possíveis erros de calibração (amplificador virtual).

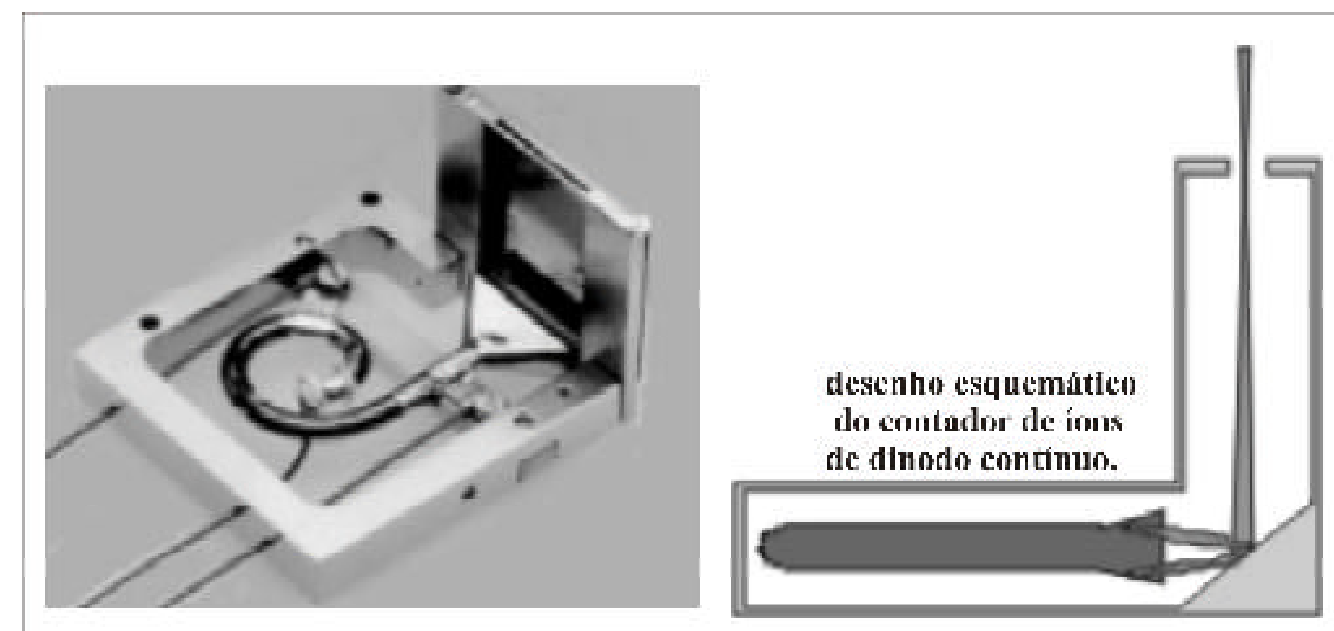

Figura 22a. Mini contador de íons utilizado no espectrômetro IsoProbe. Ref.: Micromass (2000).

Figura 22b. Mini contador de íons do NEPTUNE/TRITO N. Ref.: Finnigan Mat (2001).

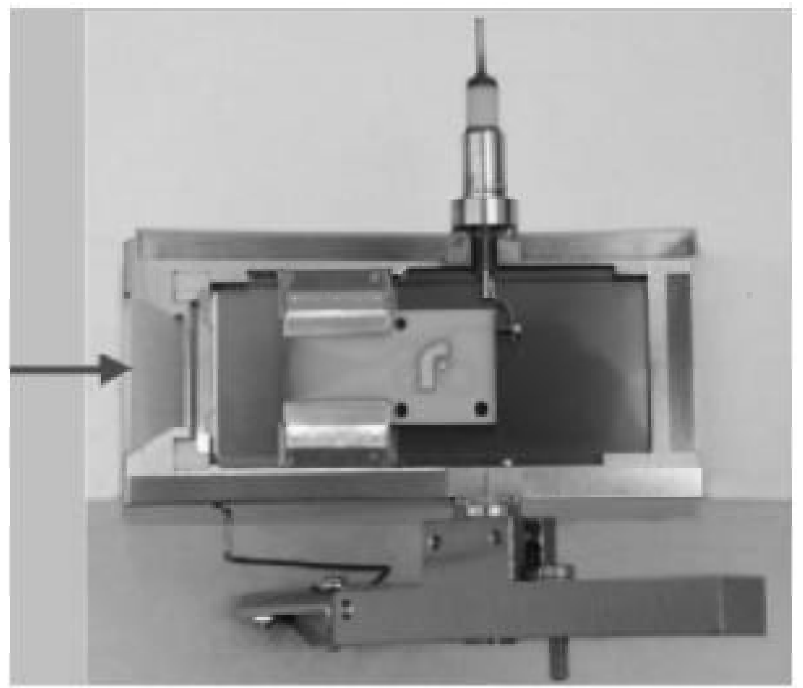




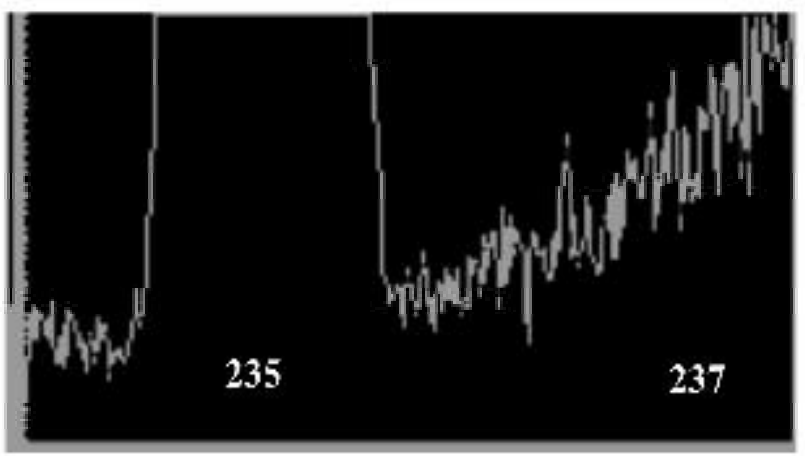

Figura 23a. "Tail" da massa 238 influenciando sobre o pico 235. Ref.: Micromass (2000).

\section{Resultados experimentais de IC P-MS-MC obtidos na "The University of $Q$ ueensland" - Brisbane - Austrália em 2000}

No laboratório "Advanced Centre for Queensland University Isotope Research Excellence" - Brisbane - Austrália, encontra-se instalado um ICP - MS de setor magnético e com sistema multicoletor da marca Micromass (IsoProbe). Os dados de razões isotópicas obtidos no laboratório acima referido em padrões de NBS e de La-Jolla, durante a visita do pesquisador do CPGeo, em Julho de 2000 podem ser vistos nas tabelas de 1 a 6 (Apêndice).

Os resultados experimentais obtidos pelo IsoProbe indicam um bom desempenho deste espectrômetro, embora alguns resultados como as razões ${ }^{143} \mathrm{Nd} /{ }^{144} \mathrm{Nd} \mathrm{e}{ }^{150} \mathrm{Nd} /{ }^{144} \mathrm{Nd}$ do padrão de La-Jolla (tabela 1), necessitem de um ajuste de "bias". Em geral, são comparáveis aos do TIMS.

\section{CO MPARAÇÕ ES EN TRE DIVERSOS TIPOS DE ESPEC TRÔ METRO S}

As comparações envolvendo TIMS, ICP-MS e SIMS podem ser resumidas da seguinte forma:

O TIMS tem uma altíssima precisão, mas em contrapartida não faz análise pontual e necessita de um laboratório químico super limpo. Quando utiliza a técnica de "step heating" em mono cristal de zircão produz exce-

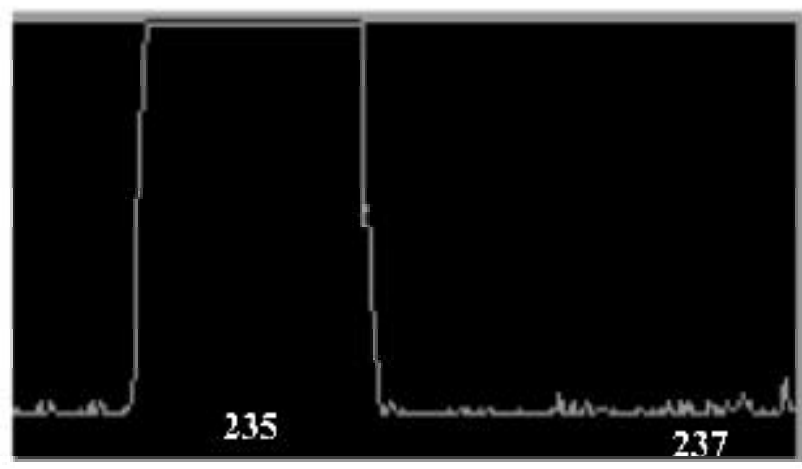

Figura 23b. Espectograma da linha base após a remoção da interferência da massa 238 que sofreu o espalhameto. Para remover a interferência foi utilizado o FILTRO WARP. Ref.: Micromass (2000).

lente resultado, desde que o cristal tenha zoneamento e sobrecrescimento regular. Portanto, a aplicação desta técnica é limitada e, além disso, o tempo analítico é grande.

O ICP - MS pode ter a precisão de TIMS se utilizar o analisador eletrostático (ESA), magnético e sistema de detecção multicoletor para análise de elemento químico em meio líquido. Para as análises envolvendo o "Laser Ablation", não é tão preciso quanto o TIMS, mas pode efetuar micro análises em um material sólido. Quando é utilizado o Laser Excimer de comprimento de onda 193 ๆm, pode-se obter análises quase comparáveis ao SHRIMP. A desvantagem na utilização do laser é a destruição do material no ponto de incidência do feixe. A utilização de abrasão a laser acoplado ao ICP-MS multicoletor de setores eletrostático e magnético ainda está em fase de teste.

Os espectrômetros de massa do tipo SIMSque utilizam a sonda iônica, tais como, o SHRIMP e CAMECA, são, no presente momento, os melhores equipamentos para efetuar datação pontual do zircão. Possui uma altíssima resolução de massa (5000 - 10000), mas é um aparelho extremamente caro. A sonda utilizando o feixe de íons de oxigênio provoca uma mini abrasão no material analisado, mas é suficiente para fazer medidas isotópicas várias vezes no mesmo ponto em dias diferentes. O erro analítico ainda é alto porque usa o monocoletor.

\section{AGRADECIMEN TOS}

Os autores agradecem a geógrafa Mitzi Sonoki pelo apoio operacional dos espectrômetros do CPGeo. Os autores são gratos aos revisores deste trabalho. 


\section{REFERÊN CIAS BIBLIOGRÁFICAS}

ALDRICH, L. T.; NIER, A. O. ( 1948) Argon 40 in potassium minerals. Physical Review, v. 74, p. 876-877.

FINNIGAN MAT (1995) Thorium isotope analyses: using the MAT 262 RPQ-II. Bremen, Germany, Finnigan Mat. (Application flash Report n. 1). (Catálogo)

FINNIGAN MAT (2001) Multicolector mass spectrometers for high precision isotope ratio determination : TRITON - NEPTUNE. Bremen, Germany, Thermo Finnigan. (Catálogo).

GBC - SCIENTIFIC EQUIPMENT PTY (1998) The advantages of time of flight mass spectrometry for elemental analysis. Australia, GBC. (Techincal Note, Publication n.01-0876-00).

HIRATA, T.; NESBIT, R. W. (1995) U-Pb isotope geochronology of zircon: evaluation of the laser probe-inductively coupled plasma mass spectrometry technique. Geochimica et Cosmochica Acta, v. 59, p. 2491-2500.

HIRATA, T.; HATTORI, M.; TANAKA, T. (1998) In situ osmium isotope ratio analyses of iridosmine by laser ablation-multiple collector - inductively coupled plasma mass spectrometry. Chemical Geology, v. 144, p. 269-280.

HIRATA, T. (2001) Isotope geochemistry. In: ANNUAL MEETING OF THE GEOCHEMICAL SOCIETY OF JAPAN, 48., Tokyo, 2001. p. S2 1-10.

HORN, I.; RUDDNICK, R. L.; MCDONOUGH, W. F. (2000) Precise elemental and isotope ratio determination by simultaneous solution nebulization and laser ablation - ICP-MS: application to U-Pb geochronology. Chemical Geology, v. 164, p. 281-300.

LI, X. H.; LIANG, X. R.; SUN, M.; GUAN, H.; MALPAS, J. G. (2001) Precise 206Pb / $238 \mathrm{U}$ age determination on zircons by laser ablation microprobe - inductively coupled plasma-mass spectrometry using continuous linear ablation. Chemical Geology, v. 175, p. 209-219.

MICROMASS (1999) Basic principles of ICP-MS IsoProbe: user's guide. Wythenshave, Micromass. p. 5-17.

MICROMASS (2000) Ion optics of multi-collector ICPMS systems for precise and accurate isotope ratio measurements. Wythenshave, Micromass. (Technical Note, n. 403).

NESBITT, R. W.; HIRATA, T.; BUTLER, I.; MILTON, J. A. (1997) UV Laser Ablation ICP-MS: some aplications in the earth sciences. Geostandards Newsletter,

$$
\text { v. } 20 \text {, n.. 2, p. 231-243. }
$$

NIER, A. O. (1939) The isotopic constitution of radiogenic leads and the measurement of geological time II. Physical Review, v. 55, p. 153-163.

NIER, A. O. (1948) Variation in the relative abundance of the isotope of common lead and the measurement of geologic time. III. Physical Review, v. 60, p. 112-116.

WILLIANS, I. S. (1998) U-Th-Pb geochronology by ion microprobe. Reviews in Economic Geology, v. 7, p.1-35. 


\section{APÊN DICE}

\begin{tabular}{|c|c|c|c|c|c|c|c|c|c|c|c|}
\hline $\begin{array}{l}\text { La- } \\
\text { Jolla }\end{array}$ & $\begin{array}{l}146 / 144 \\
\mathrm{p} / \\
\text { normalizar }\end{array}$ & $142 / 144$ & $\begin{array}{l}\text { Erro } \\
10^{-6}\end{array}$ & $143 / 144$ & $\begin{array}{l}\text { Erro } \\
10^{-6}\end{array}$ & $145 / 144$ & $\begin{array}{l}\text { Erro } \\
0^{-6}\end{array}$ & $148 / 144$ & $\begin{array}{l}\text { Erro1 } \\
0^{-6}\end{array}$ & $150 / 144$ & $\begin{array}{l}\text { Erro } \\
10^{-6}\end{array}$ \\
\hline & .7385339 & 1.141841 & $\begin{array}{l}2 \sigma \\
21\end{array}$ & .511994 & $\begin{array}{l}2 \sigma \\
6\end{array}$ & .348425 & $\begin{array}{l}2 \sigma \\
5\end{array}$ & .241565 & $\begin{array}{l}2 \sigma \\
12\end{array}$ & .236233 & $\begin{array}{l}2 \sigma \\
14\end{array}$ \\
\hline & .7385215 & 1.141851 & 23 & .511992 & 8 & .348433 & 5 & .241565 & 11 & .236223 & 15 \\
\hline & $\begin{array}{l}.7384905 \\
.7384359\end{array}$ & $\begin{array}{l}1.141854 \\
1.141869\end{array}$ & $\begin{array}{l}23 \\
37\end{array}$ & $\begin{array}{l}.512001 \\
.511989\end{array}$ & $\begin{array}{l}8 \\
10\end{array}$ & $\begin{array}{l}.348433 \\
.348428\end{array}$ & $\begin{array}{l}5 \\
10\end{array}$ & $\begin{array}{l}.241558 \\
.241570\end{array}$ & $\begin{array}{l}11 \\
20\end{array}$ & $\begin{array}{l}.236230 \\
.236239\end{array}$ & $\begin{array}{l}15 \\
21\end{array}$ \\
\hline & .7397921 & 1.141685 & 21 & .511960 & 7 & .348422 & 5 & .241530 & 9 & .236169 & 15 \\
\hline & .7398198 & 1.141689 & 16 & .511963 & 6 & .348417 & 5 & .241421 & 10 & .236165 & 12 \\
\hline & .7397517 & 1.141679 & 21 & .511960 & 7 & .348422 & 6 & .241527 & 9 & .236168 & 15 \\
\hline & $\begin{array}{l}.7397514 \\
.7397009\end{array}$ & $\begin{array}{l}1.141730 \\
1.141690\end{array}$ & $\begin{array}{l}21 \\
18\end{array}$ & $\begin{array}{l}.511971 \\
.511961\end{array}$ & $\begin{array}{l}6 \\
7\end{array}$ & $\begin{array}{l}.348422 \\
.348421\end{array}$ & $\begin{array}{l}6 \\
5\end{array}$ & $\begin{array}{l}.241527 \\
.241526\end{array}$ & $\begin{array}{l}9 \\
11\end{array}$ & $\begin{array}{l}.236168 \\
.236178\end{array}$ & $\begin{array}{l}10 \\
13\end{array}$ \\
\hline & .7396425 & 1.141711 & 21 & .511972 & 6 & .348423 & 5 & .241526 & 9 & .236174 & 12 \\
\hline & $\begin{array}{l}.7396425 \\
.7388131\end{array}$ & $\begin{array}{l}1.141802 \\
1.141816\end{array}$ & $\begin{array}{l}27 \\
34\end{array}$ & $\begin{array}{l}.511993 \\
.511992\end{array}$ & $\begin{array}{l}7 \\
8\end{array}$ & $\begin{array}{l}.348430 \\
.348424\end{array}$ & $\begin{array}{l}6 \\
7\end{array}$ & $\begin{array}{l}.241541 \\
.241535\end{array}$ & $\begin{array}{l}11 \\
11\end{array}$ & $\begin{array}{l}.236198 \\
.236190\end{array}$ & $\begin{array}{l}13 \\
15\end{array}$ \\
\hline & $\begin{array}{l}.7388363 \\
.7388455\end{array}$ & $\begin{array}{l}1.141838 \\
1.141815\end{array}$ & $\begin{array}{l}25 \\
25\end{array}$ & $\begin{array}{l}.511992 \\
.511988\end{array}$ & $\begin{array}{l}8 \\
8\end{array}$ & $\begin{array}{l}.348429 \\
.348429\end{array}$ & $\begin{array}{l}5 \\
7\end{array}$ & $\begin{array}{l}.241531 \\
.241538\end{array}$ & $\begin{array}{l}11 \\
10\end{array}$ & $\begin{array}{l}.236188 \\
.236194\end{array}$ & $\begin{array}{l}14 \\
15\end{array}$ \\
\hline & .7388398 & 1.141850 & 30 & .512002 & 7 & .348422 & 6 & .241532 & 12 & .236182 & 13 \\
\hline & $\begin{array}{l}.7388089 \\
.7388122\end{array}$ & $\begin{array}{l}1.141807 \\
1.141852\end{array}$ & $\begin{array}{l}34 \\
34\end{array}$ & $\begin{array}{l}.512004 \\
.512009\end{array}$ & $\begin{array}{l}9 \\
7\end{array}$ & $\begin{array}{l}.348441 \\
.348422\end{array}$ & $\begin{array}{l}6 \\
8\end{array}$ & $\begin{array}{l}.241538 \\
.241528\end{array}$ & $\begin{array}{l}11 \\
11\end{array}$ & $\begin{array}{l}.236200 \\
.236185\end{array}$ & $\begin{array}{l}13 \\
12\end{array}$ \\
\hline & .7387900 & 1.141860 & 27 & .512013 & 9 & .348436 & 6 & .241533 & 15 & .236178 & 15 \\
\hline $\begin{array}{l}\text { ICP-MS } \\
\text { Média }\end{array}$ & & 1.141791 & 71 & .511986 & 18 & .348427 & 6 & .241538 & 24 & .236192 & 24 \\
\hline $\begin{array}{l}* \text { TIMS } \\
\text { La-Jolla }\end{array}$ & & 1.141828 & & .511848 & & .348424 & & .241570 & & .236428 & \\
\hline $\begin{array}{l}\text { **TIMS } \\
\text { CPGeo }\end{array}$ & & 1.141840 & 30 & .511838 & 10 & .348400 & 10 & .241585 & 15 & .236460 & 15 \\
\hline
\end{tabular}

Tabela1. Razões isotópicas de N d obtidas com ICP-MS-MC ISO PRO BE no laboratório “The University of Q ueensland Austrália". O s dados acima são resultados preliminares e experimentais mostrando uma razoável concordância com - Espectrômetro de Massa de Termo-Ionização (TIMS) carecendo ainda de ajustes de fator de "bias". * Razões isotópicas de $\mathrm{Nd}$ obtidas na Universidade de California San Diego. ** Razões isotópicas de Nd, TIMS CPG eo, são as médias de dados obtidos por MAT 262 durante 0 ano base 2000 e medidas no modo estático.

\begin{tabular}{|l|l|l|ll|l|ll|}
\hline & ${ }^{176} \mathrm{Hf} /{ }^{177} \mathrm{Hf}$ & erro & ${ }^{178} \mathrm{Hf} /{ }^{177} \mathrm{Hf}$ & erro & ${ }^{180} \mathrm{Hf} /{ }^{177} \mathrm{Hf}$ & erro & No. medidas \\
\hline JM-495 & 0.28217 & $.0028 \%$ & 1.46712 & $.008 \%$ & 1.88675 & $.004 \%$ & 80 \\
\hline
\end{tabular}

Tabela 2. Razões isotópicas de Hf para uma solução de 95 ppb do padrão J M-495. O s dados acima são média de 80 razões obtidas no laboratório de "The University of Q ueensland", em Julho de 2000 . As razões de Hf foram corrigidas das interferências de Lu (5 ppb) monitorando o pico ${ }^{175} \mathrm{Lu}$. 


\begin{tabular}{|c|c|c|c|c|c|c|}
\hline NBS 983 & \multicolumn{3}{|c|}{$\begin{array}{l}\text { Julho de } 2000 \text {-The University of Queensland } \\
\text { ISOPROBE }\end{array}$} & \multirow{2}{*}{\multicolumn{2}{|c|}{$\begin{array}{l}\text { TIMS (CPGeo) - MAT262 } \\
\text { Média do ano base } 2000 \\
20 \mathrm{ng}\end{array}$}} & $\begin{array}{l}\text { Valor NBS } 983 \\
\text { certificado }\end{array}$ \\
\hline concentração & $4 \mathrm{ng} / \mathrm{g}$ & $12 \mathrm{ng} / \mathrm{g}$ & & & & \\
\hline $206 / 204$ & 0.000354 & .000370 & \pm .000003 & 0.000368 & $\pm 0,000010$ & 0,000371 \\
\hline $208 / 206$ & \pm 0.00012 & 0.01391 & \pm 0.00007 & 0.071212 & $\pm 0,000035$ & 0,071201 \\
\hline $207 / 206$ & \pm 0.00005 & 0.07132 & \pm 0.00006 & 0.013617 & $\pm .0,00008$ & 0,013619 \\
\hline
\end{tabular}

Tabela 3. Dados de razões isotópicas do padrão NBS 983 obtidos com ISO PRO BE em Julho de 2000 em The University of Q ueensland - Austrália. A análise do padrão NBS 983 com concentração super baixa de $4 \mathrm{ng} / \mathrm{g}$ mostra um desvio de $10 \%$ em relação ao valor certificado, mas o espectrômetro mostra uma sensibilidade razoável mesmo para este nível de concentração. Já as razões isotópicas de $\mathrm{Pb}$ para a concentração de $12 \mathrm{ng} / \mathrm{g}$ é comparável ao TIMS. O s dados do TIMS - CPG eo foram corrigidos usando-se um fator médio de normalização de 0,00130 u.m.a.

\begin{tabular}{|l|lllcl|}
\hline NBS 981 & \multicolumn{4}{|l}{ University of Queensland } & \multicolumn{2}{l|}{ TIMS - CPGeo / MAT262 } & NBS 981 \\
& ISOPROBE - Micromass & \multicolumn{2}{l|}{ Média do ano base 2000 } & certificado \\
& 250 razões & Erro \% & \multicolumn{3}{c}{ Erro\% } \\
$\mathbf{2 0 4 / 2 0 6}$ & 0.060746 & \pm .0009 & 0.05903 & \pm 0.02 & 0.059042 \\
\hline $\mathbf{2 0 8 / 2 0 6}$ & 2.1676 & \pm .0009 & 2.1675 & \pm 0.01 & 2.1681 \\
$\mathbf{2 0 7 / 2 0 6}$ & 0.914529 & \pm .0007 & 0.91479 & \pm 0.01 & 0.91460 \\
\hline
\end{tabular}

Tabela 4. Razões isotópicas obtidas do padrão NBS 981. A tabela mostra uma comparação entre os espectrômetros ICP-MS da ISO PRO BRE - Micromass da The University of Q ueensland e o TIMS do CPG eo. A concentração do padrão de Pb utilizado em Q ueensland foi de $50 \mathrm{ng} / \mathrm{g}$ e o número total de razões medidas foi de 250 . O s dados foram normalizados com ${ }^{203} \mathrm{TI} /{ }^{205} \mathrm{Tl}=0.41865$. Também a interferência de ${ }^{204} \mathrm{Hg}$ no ${ }^{204} \mathrm{~Pb}$ foi corrigida.

\begin{tabular}{|c|c|c|c|c|c|c|c|c|c|c|}
\hline Refer. & $206 / 204$ & $2 s$ & $207 / 204$ & $2 s$ & $208 / 204$ & $2 s$ & $207 / 206$ & $2 \mathrm{~s}$ & $208 / 204$ & $2 s$ \\
\hline \multicolumn{11}{|c|}{ ICP - MS MULTI COLETOR - ISOPROBE } \\
\hline Collerson & 16.9425 & 0.0020 & 15.4941 & 0.0021 & 36.7178 & 0.0067 & 0.91452 & 0.00004 & 2.16728 & 0.00044 \\
\hline \multicolumn{11}{|c|}{ Espectrômetro de Massa Termo Iônico (TIMS) } \\
\hline Todt* & 16.9356 & 0.0012 & 15.4891 & 0.0015 & 36.7006 & 0.0056 & 0.91459 & 0.00007 & 2.16701 & 0.00022 \\
\hline Woodhead* & 16.936 & 0.0007 & 15.492 & 0.0005 & 36.705 & 0.0011 & 0.91474 & 0.00007 & 2.16728 & 0.00022 \\
\hline Volker* & 16.937 & 0.0022 & 15.492 & 0.0025 & 36.711 & 0.0073 & 0.91468 & 0.00007 & 2.16750 & 0.00022 \\
\hline Thirlwell* & 16.9409 & 0.0011 & 15.4956 & 0.0013 & 36.7228 & 0.0040 & 0.91469 & 0.00004 & 2.16770 & 0.00011 \\
\hline Galer * & 16.9405 & 0.0011 & 15.4963 & 0.0013 & 36.7219 & 0.0040 & 0.91475 & 0.00004 & 2.16771 & 0.00011 \\
\hline CPGeo* & 16.9405 & 0.0025 & 15.4970 & 0.0035 & 36.7186 & 0.0073 & 0.91479 & 0.00009 & 2.16750 & 0.00022 \\
\hline
\end{tabular}

Tabela 5. Dados comparativos do NBS 981 analisado por ICP-MS multicoletor - ISO PRO BE (The Univeristy of Q ueensland) contra resultados por TIMS de diversos laboratórios compilados por Kenneth Collerson * apud Collerson, dados inéditos). O s dados por ICP-MS foram obtidos no período entre Maio e J unho de 2000 e as razões foram normalizadas para ${ }^{203} \mathrm{Tl} /{ }^{205} \mathrm{Tl}=0.41865$. 


\begin{tabular}{|c|c|c|c|c|c|}
\hline \multirow[t]{2}{*}{ NBS 982} & \multicolumn{2}{|c|}{$\begin{array}{l}\text { Queensland University } \\
\text { ISOPROBE - Micromass }\end{array}$} & \multicolumn{2}{|c|}{$\begin{array}{l}\text { TIMS - CPGeo / MAT } 262 \\
\text { Média do ano-base } 2000\end{array}$} & \multirow[t]{2}{*}{$\begin{array}{l}\text { Valor do NBS } 982 \\
\text { certificado }\end{array}$} \\
\hline & 350 razões & Erro \% & & & \\
\hline $204 / 206$ & 0.027194 & \pm .0010 & 0.027209 & 0.05 & 0.027219 \\
\hline $208 / 206$ & 1.00011 & \pm .0007 & 1.00008 & 0.01 & 1.00017 \\
\hline $207 / 206$ & 0.46692 & \pm .0006 & 0.46692 & 0.01 & 0.46707 \\
\hline
\end{tabular}

Tabela 6. Razões isotópicas do padrão NBS 982. A tabela mostra uma comparação entre dados por ICP-MS da ISO PRO BRE - Micromass de The University of Q ueensland e o TIMS do CPG eo. A concentração do padrão de $\mathrm{Pb}$ utilizado em Queensland foi de $50 \mathrm{ng} / \mathrm{g}$ e o número de razões medidas foi de 350. O s dados foram normalizados $\operatorname{com}^{203} \mathrm{Tl} /{ }^{205} \mathrm{TI}=0.41865$. Também a interferência de ${ }^{204} \mathrm{Hg}$ em ${ }^{204} \mathrm{~Pb}$ foi corrigida. O s dados do TIMS - CPG eo foram corrigidos usando o fator médio de normalização de 0,00130 por unidade de massa atômica (u.m.a.).

\begin{tabular}{|c|c|c|c|c|c|c|c|c|}
\hline Elem. & $\begin{array}{l}\text { Tipo de } \\
\text { filam. }\end{array}$ & $\begin{array}{l}\mathrm{M} \\
/ \mathrm{O}\end{array}$ & $\begin{array}{l}\text { Solução de } \\
\text { deposição }\end{array}$ & $\begin{array}{l}\text { Modo } \\
\text { medida }\end{array}$ & $\begin{array}{l}\text { No. } \\
\text { col. }\end{array}$ & $\begin{array}{l}\text { Razão para } \\
\text { normalização }\end{array}$ & $\begin{array}{l}\text { Interfe- } \\
\text { rência }\end{array}$ & spike \\
\hline$+\mathbf{S r}$ & $\mathrm{Ta}$ & M & $\mathrm{H} 3 \mathrm{PO} 41,0 \mathrm{~N}-2 \mu \mathrm{l}$ & $\begin{array}{l}\mathrm{PJ} \\
\mathrm{E} ; \mathrm{E} / \mathrm{D}\end{array}$ & $\begin{array}{l}1 \\
4\end{array}$ & ${ }^{86} \mathrm{Sr} /{ }^{88} \mathrm{Sr}=0,1194$ & ${ }^{87} \mathrm{Rb}$ & ${ }^{84} \mathrm{Sr}$ \\
\hline${ }^{+} \mathbf{R b}$ & Ta;Ta-Re & $\mathrm{M}$ & $\mathrm{HCl} 0,1 \mathrm{~N} ; \mathrm{H}_{2} \mathrm{O}$ & $\mathrm{PJ} ; \mathrm{E}$ & $1 ; 2$ & ----- & ${ }^{87} \mathrm{Sr}$ & ${ }^{87} \mathrm{Rb}$ \\
\hline$+\mathbf{K}$ & Ta;Ta-Re & $M$ & $\mathrm{HCl} 0,1 \mathrm{~N}$ & PJ; E & $1 ; 2$ & ---- & --- & ${ }^{40} \mathrm{~K}$ \\
\hline${ }^{+} \mathbf{C a}$ & Re-Re & M & $\mathrm{HCl} 0,1 \mathrm{~N}$ & E & 6 & ${ }^{42} \mathrm{Ca} /{ }^{44} \mathrm{Ca}=0,31221$ & ${ }^{40} \mathrm{~K}$ & ${ }^{42} \mathrm{Ca}$ \\
\hline${ }^{+} \mathbf{N d}$ & Ta-Re & $\mathrm{M}$ & $\mathrm{HCl} 0,1 \mathrm{~N}$ & $\begin{array}{l}\mathrm{PJ} ; \\
\mathrm{E} ; \mathrm{E} / \mathrm{D}\end{array}$ & $\begin{array}{l}1 \\
4\end{array}$ & ${ }^{146} \mathrm{Nd} /{ }^{144} \mathrm{Nd}=0,7219$ & ${ }^{*} \mathrm{Sm}$ & ${ }^{150} \mathrm{Nd}$ \\
\hline${ }^{+} \mathrm{NdO}$ & $\operatorname{Re}$ & $\mathrm{O}$ & $\mathrm{HNO}_{3} 0,1 \mathrm{~N}$ & $\begin{array}{l}\mathrm{PJ} ; \\
\mathrm{E} ; \mathrm{E} / \mathrm{D}\end{array}$ & $\begin{array}{l}1 \\
5\end{array}$ & ${ }^{146} \mathrm{Nd} /{ }^{144} \mathrm{Nd}=0,7219$ & $* *$ & ${ }^{150} \mathrm{Nd}$ \\
\hline${ }^{+} \mathrm{NdO}$ & $\operatorname{Re}$ & $\mathrm{O}$ & $\mathrm{H}_{3} \mathrm{PO}_{4} 0,7 \mathrm{~N}-2 \mu \mathrm{l}$ & $\mathrm{E} ; \mathrm{E} / \mathrm{D}$ & 5 & ${ }^{146} \mathrm{Nd} /{ }^{144} \mathrm{Nd}=0,7219$ & $* *$ & ${ }^{150} \mathrm{Nd}$ \\
\hline${ }^{++} \mathbf{S m}$ & $\mathrm{Ta}$ & $\mathrm{M}$ & $\mathrm{H}_{3} \mathrm{PO}_{4} 0,7 \mathrm{~N}-2 \mu 1$ & $\mathrm{PJ} ; \mathrm{E}$ & $1 ; 4$ & ${ }^{152} \mathrm{Sm} /{ }^{147} \mathrm{Sm}=1,78314$ & $* * *$ & ${ }^{149} \mathrm{Sm}$ \\
\hline$+\mathbf{P b}$ & $\operatorname{Re}$ & $M$ & $\mathrm{H}_{3} \mathrm{PO}_{4}+$ silica- $2 \mu \mathrm{l}$ & PJ; E & 6 & ${ }^{203} \mathrm{Tl} /{ }^{205} \mathrm{Tl}=0,41865$ & $\begin{array}{l}{ }^{204} \mathrm{Hg} ; \\
\text { orgânico }\end{array}$ & $\begin{array}{l}{ }^{208} \mathrm{~Pb} ; \\
{ }^{205} \mathrm{~Pb}\end{array}$ \\
\hline$+\mathbf{P b}$ & Re canoa & M & Mono cristal & PJ & 1 & ------ & $\mathrm{Pb}$ comum & ---- \\
\hline${ }^{+} \mathbf{U}$ & Re-Re; & $\mathrm{M}$ & $\mathrm{HNO}_{3} 0,1 \mathrm{~N}-2 \mu_{1}$ & PJ; E & 2 & $\begin{array}{ll}---- \\
---\end{array}$ & --- & ${ }^{235} \mathrm{U}$ \\
\hline$+\mathbf{U O}$ & $\mathrm{Re}$ & $\mathrm{O}$ & $\mathrm{H} 3 \mathrm{PO} 4$ + sílica & PJ; E & 2 & ------ & ---- & ${ }^{235} \mathrm{U}$ \\
\hline${ }^{+}$Th & $\mathrm{Re}-\mathrm{Re}$ & $\mathrm{M}$ & $\mathrm{HCl}$ & PJ; E & $1 ; 2$ & - & ---- & ${ }^{230} \mathrm{Th}$ \\
\hline$-\mathrm{OsO}_{3}$ & $\mathrm{Pt} ; \mathrm{Pt}-\mathrm{Pt}$ & $\mathrm{O}$ & $\begin{array}{l}\mathrm{Ba}(\mathrm{OH})_{2}-20 \mu \mathrm{g} ; \\
\mathrm{Ba}(\mathrm{NO} 3)_{2} ; \mathrm{Ca}(\mathrm{NO} 3)_{2}\end{array}$ & $\mathrm{PJ} ; \mathrm{E}$ & $1 ; 7$ & $\begin{array}{l}\left.{ }^{240}\left(\mathrm{OsO}_{3}\right)\right)^{236}\left(\mathrm{OsO}_{3}\right)= \\
3.092203 ; \\
{ }^{190} \mathrm{Os}^{188} \mathrm{Os}=3,08271\end{array}$ & $\begin{array}{l}{ }^{\mathrm{xxx}} \mathrm{W}^{16} \mathrm{O}_{3} \\
{ }^{187} \mathrm{Re}^{16} \mathrm{O}_{3} \\
{ }^{\mathrm{xxx}} \mathrm{Pt}^{16} \mathrm{O}_{3}\end{array}$ & ${ }^{190} \mathrm{Os}$ \\
\hline$-\mathrm{ReO}_{3}$ & $\mathrm{Pt} ; \mathrm{Pt}-\mathrm{Pt}$ & $\mathrm{O}$ & $\mathrm{Ba}(\mathrm{NO} 3)_{2}-20 \mu \mathrm{g}$ & $\mathrm{PJ} ; \mathrm{E}$ & $1 ; 2$ & ---- & ${ }^{187} \mathrm{Os}^{16} \mathrm{O}_{3}$ & ${ }^{185} \mathrm{Re}$ \\
\hline $\mathrm{BO}_{2}$ & $\operatorname{Re}$ & $\mathrm{O}$ & $\begin{array}{l}\mathrm{Na}(\mathrm{NO})_{3}-20 \mu \mathrm{g} \\
\mathrm{Ba}(\mathrm{NO} 3)_{2} ; \mathrm{Ca}(\mathrm{NO} 3)_{2}\end{array}$ & $\mathrm{PJ} ; \mathrm{E}$ & $1 ; 2$ & $\begin{array}{c}---- \\
--\end{array}$ & ---- & ${ }^{10} \mathrm{~B}$ \\
\hline
\end{tabular}

Tabela 7. Técnicas de deposição e analítica em TIMS para os principais elementos químicos utilizados em geocronologia. Legenda: $\mathbf{M}=$ metal, $\mathbf{O}=$ óxido, $\mathbf{P J}=$ "peak jump", $\mathbf{D}=$ dinâmico com multicoleção, $\mathbf{E}=$ estático. * As interferências dos isótopos de $\mathrm{BaO}$ sobre os isótopos de $\mathrm{Nd}$ são ${ }^{130} \mathrm{Ba}^{16} \mathrm{O},{ }^{130} \mathrm{Ba}^{18} \mathrm{O},{ }^{132} \mathrm{Ba}{ }^{16} \mathrm{O}$, ${ }^{132} \mathrm{Ba}^{18} \mathrm{O} \mathrm{e}^{134} \mathrm{Ba}^{16} \mathrm{O}$. ** Existem inúmeras interferências isobáricas entre os $\mathrm{NdO}$, pois o oxigênio possui 3 isótopos $\left({ }^{16} \mathrm{O},{ }^{17} \mathrm{O} \mathrm{e}{ }^{18} \mathrm{O}\right.$ ). Por exemplo, as interferências sobre a ${ }^{143} \mathrm{Nd}^{16} \mathrm{O}$ (massa total igual a 159 ) são ${ }^{141} \mathrm{Pr}^{18} \mathrm{O},{ }^{142} \mathrm{Ce}^{17} \mathrm{O}$, ${ }^{142} \mathrm{~N} \mathrm{~d}^{17} \mathrm{O}$ e as interferências isobáricas relativas à massa ${ }^{144} \mathrm{~N} \mathrm{~d}^{16} \mathrm{O}$ (massa total igual a 160 ) são ${ }^{142} \mathrm{Ce}^{18} \mathrm{O},{ }^{142} \mathrm{Nd}^{18} \mathrm{O}$, ${ }^{143} \mathrm{Nd}^{17} \mathrm{O},{ }^{144} \mathrm{Sm}^{16} \mathrm{O}$. *** O s isótopos de ${ }^{144} \mathrm{Nd},{ }^{148} \mathrm{Nd}$ e ${ }^{150} \mathrm{Nd}$ interferem diretamente sobre os isótopos de ${ }^{144} \mathrm{Sm}$, ${ }^{148} \mathrm{Sm}$ e ${ }^{150} \mathrm{Sm}$. Também os isótopos de Ba interferem em $\mathrm{Sm}$, tais como: ${ }^{130} \mathrm{Ba}^{17} \mathrm{O},{ }^{130} \mathrm{Ba}^{18} \mathrm{O},{ }^{132} \mathrm{Ba}^{17} \mathrm{O},{ }^{132} \mathrm{Ba}^{16} \mathrm{O}$, ${ }^{132} \mathrm{Ba}^{17} \mathrm{O},{ }^{132} \mathrm{Ba}^{18} \mathrm{O},{ }^{134} \mathrm{Ba}^{16} \mathrm{O},{ }^{134} \mathrm{Ba}^{18} \mathrm{O},{ }^{135} \mathrm{Ba}^{17} \mathrm{O},{ }^{136} \mathrm{Ba}^{16} \mathrm{O},{ }^{136} \mathrm{Ba}^{17} \mathrm{O},{ }^{136} \mathrm{Ba}^{18} \mathrm{O},{ }^{137} \mathrm{Ba}^{16} \mathrm{O},{ }^{137} \mathrm{Ba}^{17} \mathrm{O}$ e ${ }^{138} \mathrm{Ba}^{16} \mathrm{O}$. xxx Massas dos isótopos de W e de Pt. O bs: os óxidos de Re, O s e B são analisados na forma de íons negativos. Para isto, é necessário inverter as polarizações da tensão de aceleração e do campo magnético. 